\title{
Report on the Meeting of the International Starch Symposium in Akita, 2010 "Frontiers of New Approaches to Starch Metabolism Dynamics"
}

(J-STAGE Advance Published Date: December 6, 2011)

From November $9^{\text {th }}$ to $11^{\text {th }}$ 2010, starch scientists from Japan and around the world attended an exciting meeting in Akita in the northeast region of Japan. The Symposium had been firstly designed by Yasunori Nakamura and Naoko Fujita to provide a detailed overview of our increasing knowledge of starch biosynthesis dynamics in plants covering a wide range of materials and approaches through discussion among leading scientists. The idea for this meeting was discussed with Dr. Steven Ball when Yasunori visited Steven's lab in 2008, and we were pleased that all of our friends accepted our invitation to the meeting when presented with the idea at the Starch Round Table in Baltimore in 2009.

The International Starch Symposium in Japan focusing on starch biosynthesis was the third following the meetings in Tsukuba in 1996 and Tokyo in 2002 [see J. Appl. Glycosci., 50, 157-313 (2003)].

Nearly all the invited speakers and guests from overseas arrived in Tokyo and then transferred to Akita by domestic flights. Although flights between Tokyo and Akita are limited, all participants reached Akita on schedule.

On the first day (Tuesday, November 9), we conducted an open meeting at Akita College Plaza near Akita Station with participants gathered from every region in Japan. Each speaker presented new results (30 min) and several questions were raised, stimulating vigorous discussion and the exchange of ideas between speakers and attendants (10 min).
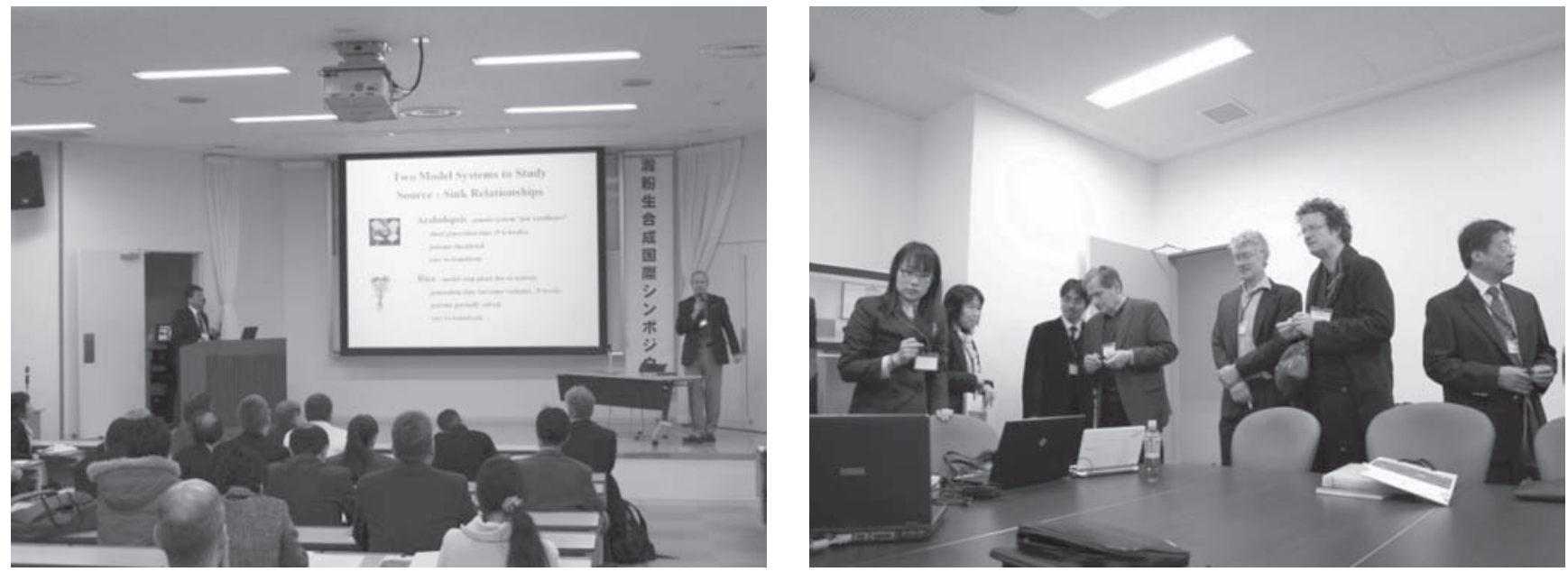

$$
==\text { Program }==
$$

International Starch Symposium in Akita-Part I

Date: November 9, 2010

Place: Akita College Plaza, Akita-city

9:00- Registration

9:20- Opening remarks (Y. Nakamura)

(chair, Yasunori Nakamura)

9:30- Isao Hanashiro (Kagoshima Univ., Japan)

"Branched structure of amylose"

10:10- Steven Ball (Univ. Sci. Tech. Lille, France)

"The evolution of the glycogen and starch pathway in eukaryotes gives molecular clues to understand the establishment of plastid endosymbiosis"

11:50- Coffee break

(chair, Mike Emes)

11:10- Thomas W. Okita (Washington State Univ., U.S.A.)

"Manipulation of starch metabolism to enhance plant productivity Using Arabidopsis and rice as Model Systems"

11:50- Matthew Morell (CSIRO, Australia)

"Synthesis of high amylose starches in barley and wheat"

12:30- Lunch

(chair, Isao Hanashiro)

13:40- Christophe D’Hulst (Univ. Sci. Tech. Lille, France) 
"On the mandatory nature of debranching for the synthesis of amylopectin"

14:20- Yasunori Nakamura (Akita Pref. Univ., Japan)

"The in vitro analysis of functions of starch synthetic enzymes from rice"

15:00- Coffee break

(chair, Steven Ball)

15:20- lan Tetlow (Univ. Guelph, Canada)

"Coordination of the enzymes of starch biosynthesis"

16:00- Alan Myers (lowa State Univ., U.S.A.)

"Maize opaque5 encodes monogalactosyldiacylglycerol synthase: Connections between starch biosynthesis and amyloplast membrane lipid composition"

16:40- Martin Steup (Univ. Potsdam, Germany)

"The phosphorylation/dephosphorylation cycles in transitory starch metabolism"

17:20 Closing remarks (N. Fujita)

17:45 Banquet (at Akita View Hotel)

Organizers: Yasunori Nakamura, Naoko Fujita

Organizing Committee: Koetsu Wabiko, Eiji Suzuki

Supporting Committee:

Tohoku Branch, Japanese Society of Applied Glycoscience

Faculty of Bioresource Sciences, Akita Prefectural University

After the meeting, a banquet was held at the Akita View Hotel beginning at $6 \mathrm{pm}$. This event was a great opportunity for many Japanese scientists to speak directly to eminent scientists from around the world and for everyone to experience local food and drinks, including the popular "sake" from the Akita area.
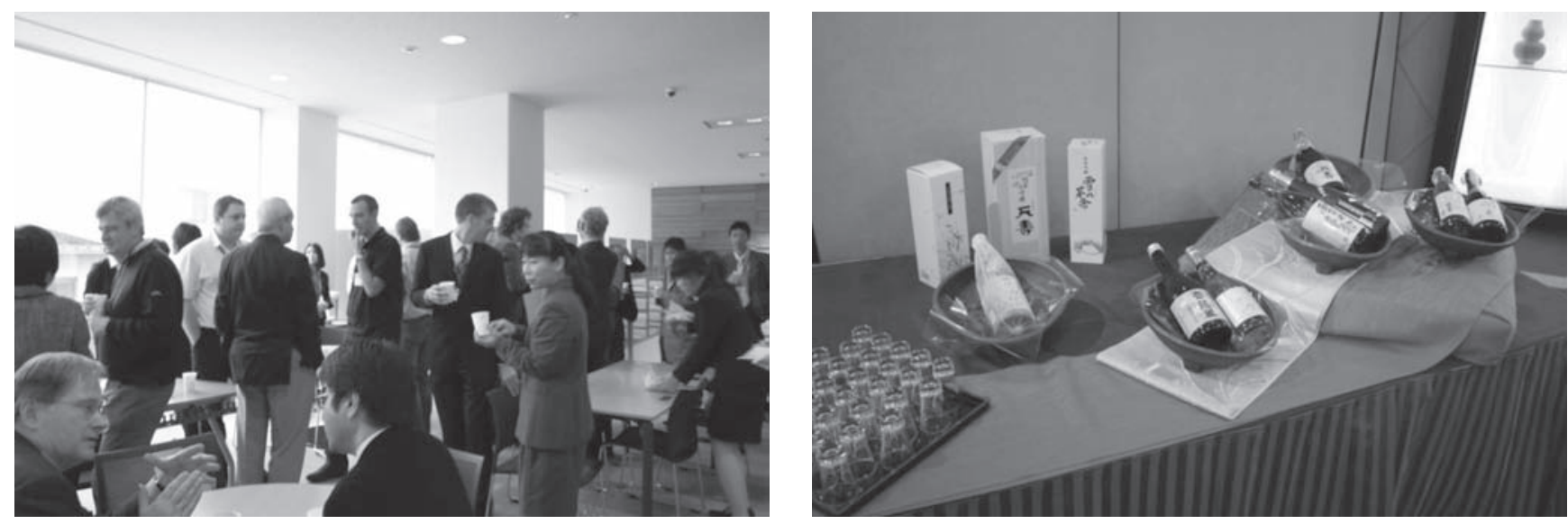

Early in the morning on the second day, we took a bus to a hotel (Hotel Kiraraka) on the Oga Peninsula for a closed symposium. During the hour-long bus tour, we enjoyed the Akita coast facing the Japan Sea, stopping at a small but beautiful beach called Unosaki Beach (see picture below).

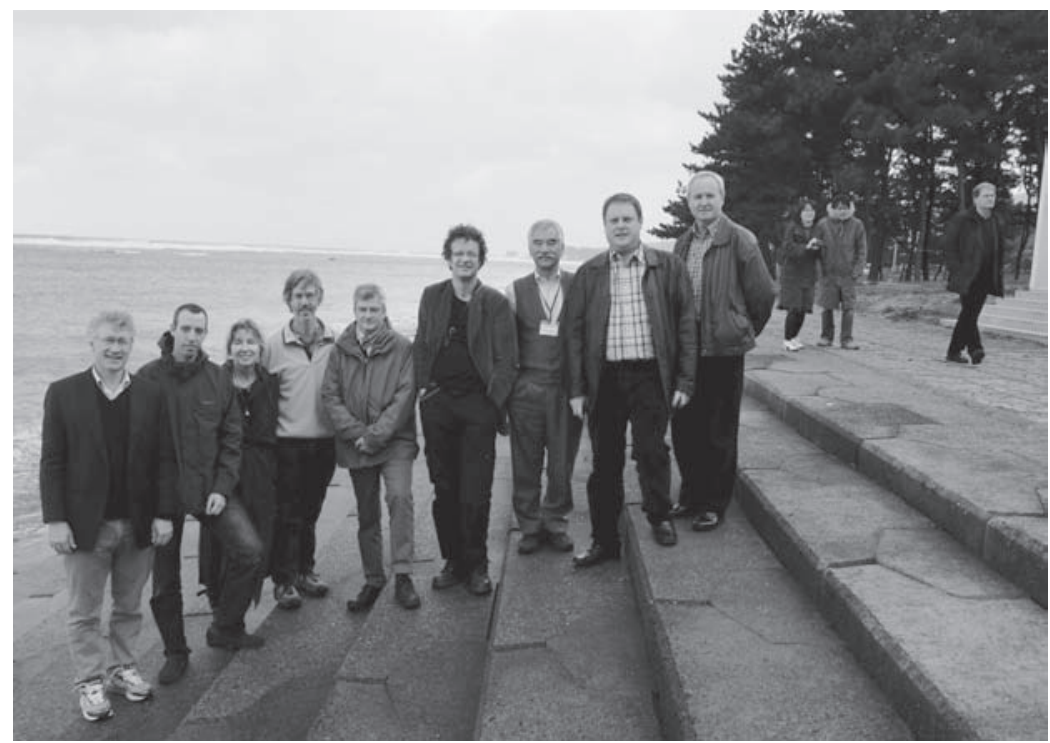

Meeting participants at Unosaki Beach. From left to right: M. Morell, F. Wattebled, C. Tirtiaux-Ball, A. Myers, C. Colleoni, S. Ball, Y. Nakamura, C. D’Hulst, M. Emes (M. Steup and the Utsumis walking on the right side of the picture). 
Soon after arriving at the hotel, we began the morning session of the second symposium at approximately 10 am and continued until approximately $5 \mathrm{pm}$. We also held a scientific session the following morning. During the second symposium, many Japanese scientists presented their recent results and invited speakers from overseas contributed to the meeting by giving additional talks.
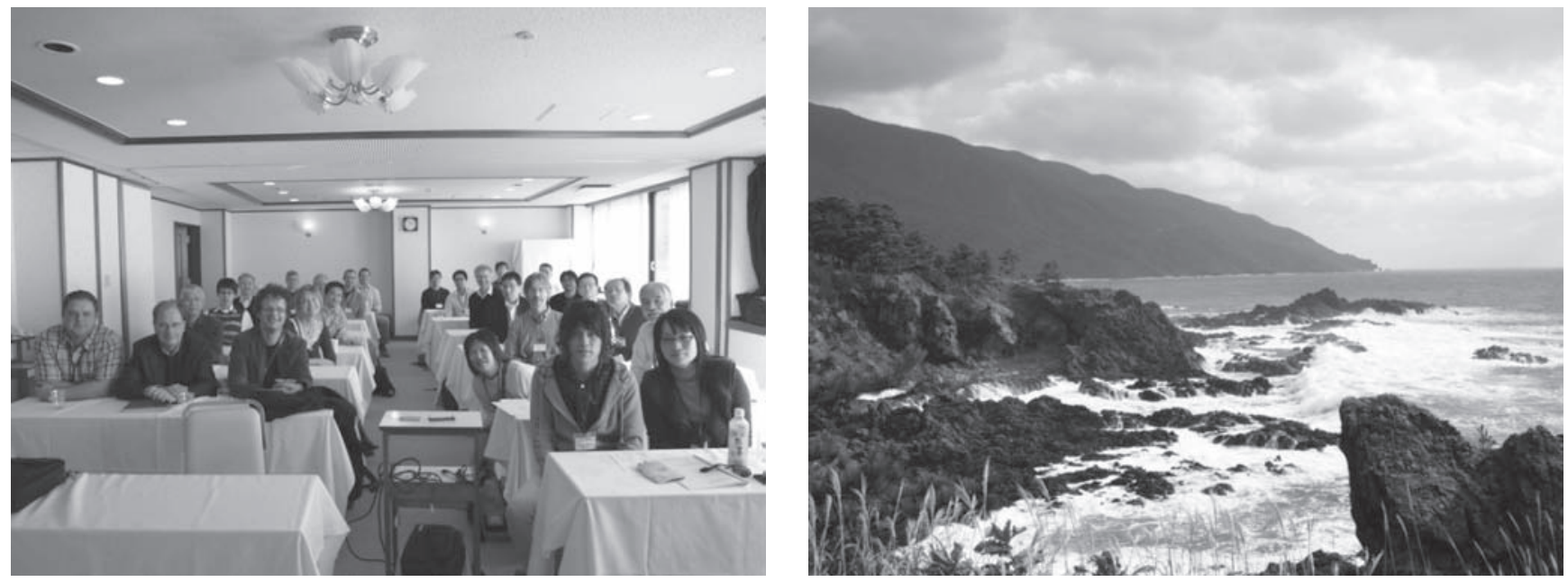

International Starch Symposium in Akita-Part II (closed meeting)

Date: November 10-11 Place: Hotel Kiraraka, Oga Peninsula

November 10

$$
==\text { Program }==
$$

9:50- Opening remarks (Y. Nakamura)

(chair, Matthew Morell)

10:00- Yasushi Kawagoe (National Institute of Agrobiological Sciences)

"Septum formation in amyloplasts produces compound granules in the rice endosperm"

10:30- Naoko Fujita (Akita Prefectural University)

"Function of starch synthase (SS) isozymes in rice endosperm"

11:00- Christophe D’Hulst (Univ. Sci. Tech. Lille)

"The mechanism underlying starch granule priming in plants: The function of Soluble Starch synthase 4 and its interconnection with the plastid division machinery"

11:30- lan Tetlow (University of Guelph)

"Regulation of starch branching enzymes"

12:00- Lunch

(chair, Martin Steup)

13:30- Hikaru Satoh (Kyushu University)

"Saturation mutant library derived from $N$-methyl- $N$-nitrosourea treatment of the fertilized egg cells in rice"

14:00- Naoyoshi Inouchi (Fukuyama University)

"Relationship between structure and physicochemical properties in rice starches"

14:30- Yasuhiro Takahata (Nat. Agric. Res. Center for Kyushu Okinawa Region)

"Analysis of starch biosynthesis in the novel sweetpotatoes that have starches pasting at low temperature"

15:00- Thomas W. Okita (Washington State University)

"Structure - function of ADPglucose pyrophosphorylase and phosphorylase and their possible interaction"

15:30- Coffee break

(chair, Christophe D'Hulst)

16:00- Hiromoto Yamakawa (National Agricultural Research Center)

"Omics-based approach for cereal starch biosynthesis: towards determination of key factors for Quality of rice grain affected by high temperature"

16:30- Steven Ball (Univ. Sci. Tech. Lille)

"Phylogenetic analysis of cristallinity determinants of starch metabolism points to the independent conversions of glycogen to starch during Archaeplastida evolution"

17:00- Christophe Colleoni (Univ. Sci. Tech. Lille)

"Characterization of a novel enzyme activity involved in semi-amylopectin biosynthesis from the marine cyanobacterium Clg1" 
17:30- Eiji Suzuki (Akita Prefectural University)

"Characterization of cyanobacterial starch"

19:00- Dinner/Banquet

\section{November 11}

(chair, lan Tetlow)

8:00- Takahiro Noda (National Agricultural Research Center)

"The effect of starch molecular structures on starch hydrothermal and degradative properties of potatoes"

8:30- Matthew Morell (CSIRO)

"Increased biomass and yield in wheat results from down regulation of glucan water dikinase activity"

9:00- Martin Steup (University of Potsdam)

"The routes of glucose 1-phosphate mediated starch biosynthesis"

9:30- Coffee break

(chair, Naoko Fujita)

10:00- Utsumi Yoshinori and Yasunori Nakamura

"Diversity of function and structure of isoamylase"

10:30- Alan Myers

"Maize SSIII affects activity of specifically the homomeric form of isoamylase-type starch debranching enzyme"

11:00- Closing remarks (N. Fujita)

11:30- Lunch

(Volunteers attended the following afternoon session as excursion)

12:30- Bus tours to Oga Peninsula and Sake Brewery (Kodama Jozo)

17:30- Dinner (Hotel Sun Rural at Ogata Village)

21:00- Akita View Hotel

At dinner, as the exciting discussion continued, we enjoyed a Japanese-style banquet at a typical hot-spring hotel found in many places in the Japanese countryside. During the banquet, we enjoyed a "Namahage" performance (picture). Next, many participated in "Karaoke," singing pop, country and other popular music.
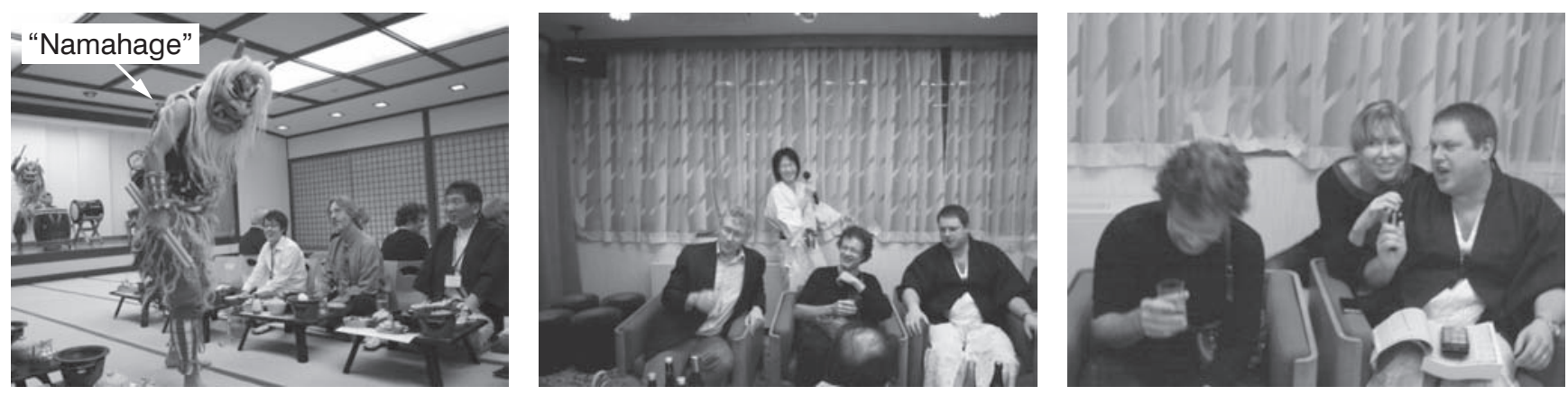

On the second day, we held discussions during the morning session. In the afternoon after closing the meeting, many participants joined a bus excursion. First, they visited the top of the Oga Peninsula called "Nyudosaki," followed by the Sake brewery "Kodama Jozo" where they learned how Japanese sake is brewed. The final leg of the excursion was dinner at a Japanese French Restaurant in a hotel in Ogata Village. This area was previously a large lake but is now a very large rice paddy field with an average height of about $4 \mathrm{~m}$ below sea level.

An impression of the meeting was presented by Dr. Fabrice Wattebled as follows:

An international symposium devoted to the presentation of recent progress made in the field of starch metabolism was organized in Akita, Japan by Drs. Yasunori Nakamura and Naoko Fujita and his collaborators at the Akita Prefectural University. During the conference, scientists originating from different countries (Australia, Canada, France, Germany, USA and Japan) shared their most recent results in a friendly atmosphere. Considerable new data regarding different steps in starch metabolism were presented. A variety of organisms (potato, sweetpotato, cereals, Arabidopsis, microalgae and cyanobacteria) and approaches used by scientists in their research revealed new findings in different fields, including the following:

(1) The evolution of storage polysaccharide metabolism.

(2) Fundamental aspects: enzymes function, protein-complexes regulations, etc.

(3) Industrial applications: increasing plant yield and modification of starch structure and properties.

We learned the most recent emerging hypothesis attempting to explain how starch was selected through evolution as a 
hydrosoluble and amorphous storage polysaccharide, and how this process can be used to explain the establishment of plastid endosymbiosis when cyanobacteria were engulfed by eukaryotic hosts. Studies involving starch-accumulating cyanobacteria and further phylogenetic analysis of protein sequences from diverse sources (now available because of the ever-increasing number of fully sequenced genomes) provided new insights supporting these ideas.

The relationship between starch metabolism and plastid physiology appears closer than first hypothesized. In rice, septum formation in the amyloplast modifies granule morphology, leading to the formation of compound granules. In Arabidopsis, a starch-metabolizing enzyme (Soluble Starch synthase 4) appears interconnected with plastid division machinery. Finally, evidence of connections between starch biosynthesis and amyloplast membrane lipid composition were highlighted.

During the meeting, new developments were presented concerning the various steps in starch metabolism. The catalytic properties of several starch-synthesizing enzymes, including branching enzymes and starch synthases, have been determined by in vitro analyses of recombinant or native enzymes (in rice). Studies performed in wheat showed that most of these enzymes are involved in the formation of huge protein complexes and that post-translational modifications such as phosphorylation are required.

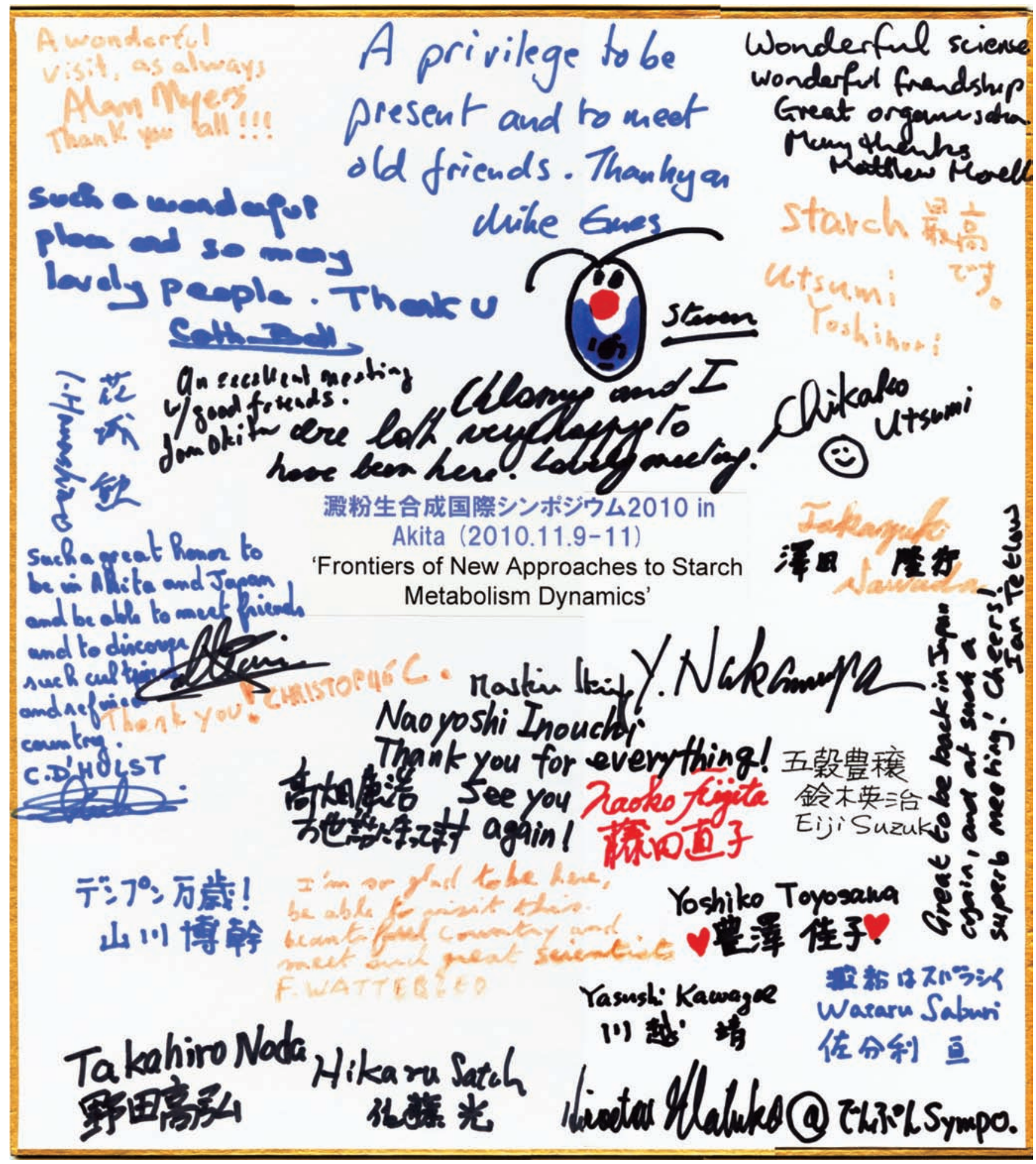


Several presentations were devoted to debranching catalyzed by isoamylases (or pullulanase). In rice, it was reported for the first time that the heteromeric complex involved in anabolism is carried out by a complex composed of five ISA1 and one ISA2 subunits. In Arabidopsis, in vivo analyses performed using single, double, or triple mutant lines highlighted the overlapping functions among the three different activities in the anabolic or catabolic steps of starch metabolism.

A full session of the meeting was dedicated to applied research. First, a saturated mutant library of rice engendered by chemical agents that can produce point mutations and non-GMO plants was presented. There is no doubt that such a tool will allow our colleagues studying rice to create new and interesting mutants to improve our understanding of starch metabolism and regulation.

Next, presentations explained how plant yield has been increased through higher leaf starch accumulation, and how higher grain yield in wheat has been achieved through down-regulation of a single enzyme involved in starch degradation (GWD). This finding was surprising since the control of grain yield was suspected to be a multigenic trait in cereals.

This meeting also provided the opportunity for some participants to discover Japanese traditions and refined culture; Akita is an average-sized city located northwest of Honshu Island facing the Japanese Sea at the $40^{\text {th }}$ north parallel (the same latitude as Madrid, Ankara, New York and Beijing).

We were delighted by traditional dishes served in a traditional Japanese style and atmosphere (it was challenging for some of us to sit directly on the floor at dinner!). We sampled various brands of sake and compared them. After several glasses, we watched three Namahages playing taiko, dancing, and screaming in front of us (trying to scare us as they typically do with children that are not well-behaved at home). After a few more glasses of sake, we were ready for a terrific karaoke session! Fortunately, no one besides meeting participants was present in the hotel to hear us singing traditional Japanese, English, American and French songs!!!

All meeting-participants were grateful to the organizers, Yasunori, Naoko, Eiji and other colleagues for the very good job they did during these days and for the exciting science and enjoyable atmosphere during our visit to Japan. Arigato! Mata oai shimasho (See you again soon)!

Overall, we all felt the same as Steven regarding the meeting: “The meeting was indeed a wonderful experience. Quite frankly, the quality of the meeting was extremely high since people were not afraid of discussing unpublished data in great detail. The location on the Oga Peninsula was spectacular." As organizers, we thank all participants for sharing exciting moments and for their great cooperation and contributions. We appreciate the e-mail messages received after the symposium; for example, from Dr. Mike Emes, “Thank you so much for making the Starch Symposium such an outstanding success. It was a great opportunity to discuss progress in the field, to remark old acquaintances, and also to establish new ones. The tone of the meeting was very open and engaging, which has much to do with the quality of the organization. The accommodation was excellent, the food magnificent and the karaoke and sake - wee, what can I say?"

Finally, we again express our thanks to invited speakers and guests from overseas and the many Japanese speakers, participants and staff for their great contribution and assistance with the symposium. We are also grateful for the assistance provided by The Tohoku Branch of The Japanese Society of Applied Glycoscience and Akita Prefectural University. This meeting was funded by grants to Y.N. from the Japan Society for the Promotion of Science (20248002), to N.F. from the program for the Promotion of Basic and Applied Research for Innovations in Bio-oriented Industry and from Akita Prefectural University.

Yasunori Nakamura

Naoko Fujita

(Organizers of the Symposium) 


\section{Abstracts for Open Symposium}

\section{Branched Structure of Amylose}

\author{
Isao Hanashiro ${ }^{1, *}$ \\ ${ }^{1}$ Faculty of Agriculture, Kagoshima University \\ (1-21-24 Korimoto, Kagoshima 890-0065, Japan)
}

Amylose is one of the 2 major $\alpha$-glucan components of native starch granules. It is well-known to greatly affect the functional properties of starch, but its structural characteristics have not been well studied. Amylose is often described as a "linear" molecule, in contrast to amylopectin, which is a highly branched molecule. The linearity of amylose implies the importance of "branched" amylose structures; it is expected that even a small number of branches on linear molecules confer large effects on the behavior of amylose molecules. However, many aspects of branched amylose structures remain unknown, including side-chain distributions and incomplete debranching by bacterial debranching enzymes. One reason for the limited knowledge is the lack of practical effective analytical methods for studying these properties.

In this study, methods of analyzing branched amylose structures have been developed, employing enzymatic debranching followed by fluorescent labeling/high-performance size-exclusion chromatography (HPSEC). The method allows differential measurement of size distributions of main and side chains of branched molecules. Main-chain distribution was determined using HPSEC of debranched amylose labeled before debranching. In addition, by labeling after debranching, both main and side chains were detected. Side-chain distribution was obtained by subtracting the detector response of the main chain from the response of main and side chains. Main chains showed only a slight difference in size distribution from that of amylose. As reported previously, debranching of amylose was incomplete, and the degree of debranching was increased by prior hydrolysis with $\beta$-amylase. Side chains released by debranching enzymes were divided into long and short chains, and the distribution for the latter was similar to that of the amylopectin counterpart from the same starch. Despite these similarities, chain-length distributions of $\beta$-amylase limit dextrin were distinct between amylose and amylopectin. The molar ratio of A and B chains was significantly higher and lower, respectively, in amylose than in amylopectin. This implies that unlike amylopectin, most short side chains of amylose are not organized in a cluster structure.

*E-mail: ihanashi@chem.agri.kagashima-u.ac.jp $* * * * * * * * * *$

\section{The Evolution of the Glycogen and Starch Pathway in Eukaryotes Gives Molecular Clues to Understand the Establishment of Plastid Endosymbiosis}

\author{
Steven Ball, ${ }^{1, *}$ Christophe Colleoni ${ }^{1}$ \\ and Jenifer Nirmal Raj ${ }^{1}$ \\ ${ }^{1}$ Unité de Glycobiologie Structurale et Fonctionnelle, \\ UMR 8576 CNRS-USTL, Bâtiment C9 \\ (Cité Scientifique, 59655 Villeneuve d'Ascq, France)
}

Solid semi-crystalline starch and hydrosoluble glycogen define two distinct physical states of the same type of storage polysaccharide. Appearance of semi-crystalline storage polysaccharides appears linked to the requirement of unicellular diazotrophic cyanobacteria to fuel nitrogenase and protect it from oxygen through respiration of vast amounts of stored carbon.

Starch metabolism itself resulted from the merging of the bacterial and eukaryote pathways of storage polysaccharide metabolism after endosymbiosis of the plastid. This generated the three Archaeplastida lineages: the green algae and land plants (Chloroplastida), the red algae (Rhophyceae) and the glaucophytes (Glaucophyta).

Reconstruction of starch metabolism in the common ancestor of Archaeplastida suggests that polysaccharide synthesis was ancestrally cytosolic. In addition the synthesis of cytosolic starch from the ADP-glucose exported from the cyanobacterial symbiont possibly defined the original metabolic flux by which the cyanobiont provided photosynthate to its host. Additional evidence supporting this scenario include the monophyletic origin of the major carbon translocators of the inner membrane of eukaryote plastids which are sisters to nucleotide-sugar transporters of the eukaryote endomembrane system. It also includes the extent of enzyme subfunctionalization that came as consequence of the rewiring of this pathway to the chloroplasts in the green algae. Recent evidence suggest that at the time of endosymbiosis obligate intracellular energy parasites related to extant Chlamydia have donated genes which were critical for establishment of this ancestral starch metabolism flux.

*E-mail: steven.ball@univ-lille1.fr $* * * * * * * * * *$

\section{Manipulation of Starch Metabolism to Enhance Plant Productivity Using Arabidopsis and Rice as Model Systems}

\author{
Thomas W. Okita, ${ }^{1, *}$ Kelly Gibson, ${ }^{1}$ Yasuko Nagai, ${ }^{1}$ \\ Seon-Kap Hwang, ${ }^{1}$ Aytug Tuncel, ${ }^{1}$ Gerald Edwards, ${ }^{1,2}$ \\ Jong-Sug Park, ${ }^{3}$ Hiroyuki Ito ${ }^{4}$ and Hikaru Satoh ${ }^{5}$ \\ ${ }^{1}$ Institute of Biological Chemistry and \\ ${ }^{2}$ School of Biological Sciences, Washington State University \\ (Pullman, Washington 99164 USA) \\ ${ }^{3}$ National Academy of Agricultural Science, RDA \\ (Suwon 44-707, Korea) \\ ${ }^{4}$ Department of Chemical and Biological Engineering, \\ Akita National College of Technology \\ (1-1 Iishimabunkyou-cyo, Akita 011-8511, Japan) \\ ${ }^{5}$ Laboratory of Genetic Resources, Faculty of Agriculture, \\ Kyushu University \\ (Hakozaki 6-10-1, Fukuoka 812-8581, Japan)
}

Plant productivity is based on source-sink relationships, i.e. the capacity of source leaves to fixed $\mathrm{CO}_{2}$ to form sugars, amino acids and other metabolites and the capacity to utilize these newly synthesized compounds by non-photosynthetic sink organs for growth and development. Since relatively minor increments in the efficiency of photosynthesis can result in elevated productivity, considerable research effort is directed towards altering the components of the photosynthetic apparatus and $\mathrm{CO}_{2}$ fixation. In addition to the 
photochemical-biochemical events of photosynthesis, however, an alternative and equally viable approach to increase productivity is to maximize the utilization of newly synthesized photosynthate in leaves and in storage organs.

In many plants, starch is the dominant reserve in leaves and sink organs. The important of leaf starch as a storage reserve is best exemplified by the properties of Arabidopsis plants possessing varying capacities to synthesize leaf starch. ${ }^{1)}$ A null mutant for leaf starch grows slower and exhibits lower rates of $\mathrm{CO}_{2}$ assimilation and $\mathrm{O}_{2}$ evolution under saturating light and $\mathrm{CO}_{2}$ than a starch deficient (40\% of normal) line which, in turn, grows slower and displays lower photosynthetic properties than wild type. Moreover, the starch mutant plants show a loss of $\mathrm{O}_{2}$ sensitivity indicating photosynthetic feedback due to decrease potential to utilize triose-phosphate when photorespiration is lowered. These results suggest that efficient synthesis of leaf starch synthesis is essential for optimal photosynthesis as a means to recycle inorganic phosphate for ATP synthesis and ribulose bisphosphate regeneration. Hence, increasing leaf starch synthesis may enhance photosynthetic capacity by recycling Pi more efficiently.

To test this hypothesis, Arabidopsis TL46 line, which contains a null mutation in the AGL1 gene (codes for the leaf ADPglucose pyrophosphorylase [AGPase] large subunit) was complemented with wild type and three up-regulated Agl1 genes. Expression of the wild type AGL1 transgene restored normal leaf starch metabolism, plant growth and $\mathrm{CO}_{2}$ assimilation capacity. Expression of the upregulated AGL1 genes resulted in elevated leaf starch accumulation and turnover due to increased AGPase activity. Moreover, these transgenic plants showed enhanced rates of $\mathrm{CO}_{2}$ assimilation and growth compared to wild type. These results indicate that leaf starch is essential for optimal photosynthetic capacity and, in turn, growth and development in Arabidopsis. Similar studies in rice, a poor leaf starch accumulator $^{2,3)}$ and prone to photosynthetic feedback, ${ }^{4}$ indicated that increases in leaf starch production and turnover improved the photosynthetic, growth and yield properties.,6) Enhanced biomass production is also evident in lettuce engineered for enhanced starch production in leaves. ${ }^{7)}$

In addition to leaf starch, we have engineered starch production in developing rice seeds via the expression of a bacterial AGPase gene $g l g C-T M{ }^{8}{ }^{8}$ Expression of the bacterial AGPase in the cytoplasm resulted in increases in starch synthesis and, in turn, seed weight (up to 11\%). Curiously, transgenic expression of the potato AGPase Upreg1 gene resulted in higher seed yields due to increases in the number of panicles and grains/panicle but not in individual grain weight. ${ }^{6)}$ Stimulation of $\mathrm{CO}_{2}$ assimilation rates did not further increase grain weight in the $g l g C-T M$ expressing plants, indicating that the AGPase catalyzed step was no longer limiting starch production. Metabolite studies showed that ADPglc levels were elevated $29-46 \%$ in the transgenic lines, a level substantially larger than the net increases (7-13\%) in seed weight. ${ }^{9)}$ Interestingly, precursors leading to ADPglc formation were also elevated. These results confirmed the conclusion that ADPglc synthesis was no longer limiting and that carbon flow into starch was constrained at one or more downstream processes. Current efforts are being directed at identifying the processes that limit carbon flow into starch in these transgenic rice plants.

\section{REFERENCES}

1 ) J. Sun, T.W. Okita and G.E. Edwards: Modification of carbon partitioning, photosynthetic capacity, and $\mathrm{O}_{2}$ sensitivity in Arabidopsis plants with low ADP-glucose pyrophosphorylase activity. Plant Physiol., 119, 267-276 (1999).

2 ) H. Nakano, A. Makino and T. Mae: Effects of panicle removal on the photosynthetic characteristics of the flag leaf of rice plants during the ripening stage. Plant Cell Physiol., 36, 653-659 (1995).

3 ) H. Nakano, A. Makino and T. Mae: The effect of elevated partial pressures of $\mathrm{CO}_{2}$ on the relationship between photosynthetic capacity and N content in rice leaves. Plant Physiol., 115, 191198 (1997).

4 ) J. Sun, T.W. Okita and G.E. Edwards: Feedback inhibition of photosynthesis in rice measured by $\mathrm{O}_{2}$ dependent transients. Photosynth. Res., 59, 187-200 (1999).

5 ) K. Gibsob, J.-S. Park, Y. Nagai, Y.-C. Choi, K.-H. Roh, S.-M. Lee, D.-H. Kim, S.-B. Choi, H. Ito, G.E. Edwards and T.W. Okita: Exploiting leaf starch synthesis as a transient sink to elevate photosynthesis, plant productivity and yields. Plant Sci.,181: 275-281 (2011).

6 ) S.M. Lee, Y.H. Lee, H.U. Kim, S.C. Seo, S.J. Kwon, H.S. Cho, S.I. Kim, T. Okita and D. Kim: Characterization of the potato upreg1 gene, encoding a mutated ADP-glucose pyrophosphorylase large subunit, in transformed rice. Plant Cell Tiss. Organ Cult., 102, 171-179 (2010).

7 ) S.-M. Lee, T.-H. Ryu, S.-I. Kim, T. Okita and D. Kim: Kinetic and regulatory properties of plant ADP-glucose pyrophosphorylase genetically modified by heterologous expression of potato upreg mutants in vitro and in vivo. Plant Cell Tiss. Organ Cult., 96, 161-170 (2009).

8 ) C. Sakulsingharoj, S.-B. Choi, S.-K. Hwang, J. Bork, C.R. Meyer, G.E. Edwards, J. Preiss and T.W. Okita: Engineering starch biosynthesis for enhanced rice yields: the role of the cytoplasmic ADP-glucose pyrophosphorylase. Plant Sci., 167, 1323-1333 (2004).

9 ) Y.S. Nagai, C. Sakulsingharoj, G.E. Edwards, H. Satoh, T.W. Greene, B. Blakeslee and T.W. Okita: Control of starch synthesis in cereals: Metabolite analysis of transgenic rice expressing an up-regulated cytoplasmic ADP-glucose pyrophosphorylase in developing seeds. Plant Cell Physiol., 50, 635-643 (2009).

*E-mail: Okita@wsu.edu

\section{Synthesis of High Amylose Starches in Barley and Wheat}

\author{
M.K. Morell, ${ }^{1,2, *}$ A. Regina, ${ }^{1,2}$ S. Jobling ${ }^{1,2}$ and Z. Li $^{1,2}$ \\ ${ }^{1}$ CSIRO Food Futures Flagship \\ (PO Box 93, North Ryde NSW 1670, Australia) \\ ${ }^{2}$ CSIRO Plant Industry \\ (GPO Box 1600, Canberra, ACT 2601, Australia)
}

The biological role of starch as a key polysaccharide reserve in plants, algae, and cyanobacteria provide a significant justification for understanding the biosynthetic mechanisms responsible for its synthesis and degradation. However, the increasing realisation of the important role that complex carbohydrates play in promoting human health through the diet provides a compelling case for the better understanding of starch synthesis, structure and function given the relationships between starch consumption, type II diabetes, colorectal cancer and bowel health. High amylose starches represent the predominant mechanism for delivering slowly digested starches through the food chain.

Three potential mechanisms for increasing the apparent 
amylose content of cereals have been described. Firstly, over-expression of granule-bound starch synthase leading to elevation of amylose content has been described, but has yet to be shown to lead to amylose contents leading to high resistant starch contents. Secondly, reduction in starch branching enzyme activity has been shown in maize, wheat, rice and barley to lead to a high amylose phenotype, however, there are important differences between species in the control of amylose content by individual members of the branching enzyme gene family. Thirdly, high apparent amylose contents can be generated by decreasing amylopectin synthesis through reduction in starch synthase activity.

In this presentation we describe novel genetic mechanisms for increasing amylose content and the potential for resistant starch formation in barley and wheat through the down regulation of multiple starch synthesis genes. The differential starch structural and functional properties of these defined genotypes are described.

* E-mail: Matthew.Morell@csiro.au $* * * * * * * * * *$

\section{On the Mandatory Nature of Debranching for the Synthesis of Amylopectin}

\author{
Fabrice Wattebled, ${ }^{1}$ Ying Dong, ${ }^{1}$ Sylvain Dumez, ${ }^{1}$ \\ Veronique Planchot, ${ }^{2}$ Nocolas Szydlowski, ${ }^{1}$ \\ Bruno Pontoire, ${ }^{2}$ Aline Devin, ${ }^{1}$ Steven Ball ${ }^{1}$ \\ and Christophe D'Hulst ${ }^{1, *}$ \\ ${ }^{1}$ Unité de Glycobiologie Stucturale et Fonctionnelle, \\ UMR8576 CNRS/Univ Lille 1 \\ (59655 Villeneuve d'Ascq Cedex France) \\ ${ }^{2}$ Unité de Recherche Biopolymères Interactions Assemblages, \\ INRA de Nantes \\ (44316 Nantes Cedex 3, France)
}

Four isoforms of starch debranching enzymes (DBEs) are found in the genome of Arabidopsis thaliana: three isoamylases (ISA1, ISA2 and ISA3) and a pullulanase (PU1). DBEs are hydrolyzing enzymes that cleave $\alpha-(1 \rightarrow 6) O$-glycosidic bonds between glucose residues of starch polymers. Isoamylases and pullulanase differ from one another at both biochemical and phylogenetics levels. We have selected mutant lines defective for each of the four (DBE) genes (AtISA1, AtISA2, AtISA3 and AtPU1) and we have analyzed their ability to synthesize starch., ${ }^{1,2)}$ Our results indicate that both ISA1 and ISA2 are required for the production of a functional isoamylase-type of DBE named Iso1, the major isoamylase activity found in leaves. The absence of Iso1 leads to an $80 \%$ decrease in the starch content in both lines and to the accumulation of water-soluble polysaccharides whose structure is similar to glycogen. In addition, the residual amylopectin structure (at the level of glucan length distribution) in the corresponding mutant lines displays a strong modification when compared to the wild type, suggesting a direct, rather than an indirect, function of Iso1 during the synthesis of amylopectin and, by consequence, the synthesis of starch. This result invalidates the WSP clearing model suggested by Zeeman et al. ${ }^{3)} 1998$. Conversely it validates the amylopectin trimming model proposed by Ball et al. ${ }^{4)} 1996$ and refined by Myers et al.5) 2000. This model implies the direct implication of isoamylase in the building of the amylopectin crystal. The function of the "synthetic isoamylase" is then to allow the maturation of the structure of a precursor molecule of amylopectin (called pre-amylopectin) through the removing of exceeding branches $(\alpha-(1 \rightarrow 6)$ $O$-glycosidic bonds) introduced by branching enzymes.

On the other hand, mutants carrying a defect in the structural gene of ISA3 display a starch-excess phenotype at the end of both the light and the dark phases. This increase comes with a minor modification of the distribution of the amylopectin-forming glucans. This result suggests that this isoamylase-type of DBE plays a major role during starch mobilization.

Finally, the analysis of the pu1- mutant did not lead to a clear-cut phenotype. However, the isa2- pu1- double-mutant line displays a $92 \%$ decrease in starch content. This suggests that the function of pullulanase partly overlaps that of Iso1, although its implication remains negligible when Iso1 is present within the cell.

To go further in our understanding of the function of each isoform in the starch pathway, and because starch-like (insoluble and crystalline) material is still produced even in the absence of Iso1 we wanted determined the levels of redundancy existing between these isoforms of DBE. To this end we have produced and analyzed different combinations of mutations including isa3-1 pu1-1, isa1-1 isa3-1 and isa1-1 isa3-1 pu1-1. The latter mutant was completely devoid of DBE activity. While the starch content was strongly increased in the isa3-1 pu1-1 double mutant, it was decreased by over 98\% in the isa1-1 isa3-1 genotype and almost vanished in triple mutant combination. In addition, whereas the isa3-1 pu1-1 double mutant synthesized starch very similar to that of the wild type, the structure of the residual starch present either in isa1-1 isa3-1 or in isa1-1 isa3-1 pu1-1 combination was deeply affected. In the same way, water-soluble polysaccharides that accumulate in the isa1-1 isa3-1 and isa1-1 isa3-1 pu1-1 genotypes display strongly modified structure compared to those found in isa1-1. Taken together, these results show that in addition to its established function in polysaccharide degradation, the activity of ISA3 is partially redundant to that of Iso1 for starch synthesis. Our results also reveal the dual function of pullulanase since it is partially redundant to ISA3 for degradation and to ISA1 for synthesis. Moreover X-ray diffraction analyses suggest that the crystallinity and the presence of the 9- to $10-\mathrm{nm}$ repetition pattern in starch precisely depend on the level of debranching enzyme activity.

Finally, we wanted to evaluate the contribution of SSIV ${ }^{6,7)}$ for the priming of residual starch and phytoglycogen in the isa1- or the isa2- mutant. Thus, ss4- isa1- and ss4- isa2double mutants were engendered and analyzed. Surprisingly, the accumulation of both residual starch and phytoglycogen was not affected in the double mutants in comparison to the isa1- and isa2- mutants. New investigations are in progress to determine the impact of starch and phytoglycogen structures.

\section{REFERENCES}

1 ) F. Wattebled, Y. Dong, S. Dumez, D. Delvallé, D. Planchot, P. Berbezy, D. Vyas, P. Colonna, M. Chatterjee, S. Ball and C. D'Hulst: Mutants of Arabidopsis lacking of chloroplastic isoamylase accumulate phytoglycogen and an abnormal form of amylo- 
pectin. Plant Physiol., 138, 184-195 (2005).

2 ) F. Wattebled, V. Planchot, Y. Dong, N. Szydlowski, B. Pontoire, A. Devin, S. Ball and C. D'Hulst: Further evidence for the mandatory nature of polysaccharide debranching for the aggregation of semicrystalline starch and for overlapping functions of debranching enzymes in Arabidopsis leaves. Plant Physiol., 148, 13091323 (2008).

3 ) S.C. Zeeman, F. Northrop, A.M. Smith and T. Ap Rees: A starchaccumulating mutant of Arabidopsis thaliana deficient in a chloroplastic starch-hydrolysing enzyme. Plant Cell, 10, 16991712 (1998).

4 ) S. Ball, H.P. Guan, M. James, A. Myers, P. Keeling, G. Mouille, A. Buléon, P. Colonna and J. Preiss: From glycogen to amylopectin: A model for the biogenesis of the plant starch granule. Cell, 86, 349-352 (1996).

5 ) A.M. Myers, M.K. Morell, M.G. James and S.G. Ball: Recent progress toward understanding biosynthesis of the amylopectin crystal. Plant Physiol., 122, 989-998 (2000).

6 ) I. Roldán, F. Wattebled, M.M. Lucas, D.V. Planchot, S. Jiménez, R. Pérez, S. Ball, C. D’Hulst and Á. Mérida: The phenotype of soluble starch synthase IV defective mutants of Arabidopsis thaliana suggests a novel function of elongation enzymes in the control of starch granule formation. Plant J., 49, 492-504 (2007).

7 ) N. Szydlowski, P. Ragel, S. Raynaud, M.M. Lucas, I. Roldán, M. Montero, F.J. Muñoz, M. Ovecka, A. Bahaji, V. Planchot, J. Pozueta-Romero, C. D’Hulst and Á. Mérida: Starch granule initiation in Arabidopsis requires the presence of either class IV or class III starch synthases. Plant Cell, 21, 2443-2457 (2009).

* E-mail: Christophe.dhulst@univ-lille1.fr $* * * * * * * * * *$

\section{In vitro Analysis of Functions of Starch Synthetic Enzymes from Rice}

\author{
Yasunori Nakamura, ${ }^{1,2, *}$ Takayuki Sawada ${ }^{2}$ \\ and Naoko Fujita ${ }^{1}$ \\ ${ }^{1}$ Department of Biological Production, Faculty of \\ Bioresource Sciences, Akita Prefectural University \\ ${ }^{2}$ Biotechnology Center, Faculty of Bioresource Sciences, \\ Akita Prefectural University \\ (241-438 Kaidobata-nishi, Shimoshinjo-nakano, \\ Akita 010-0195, Japan)
}

Plant amylopectin has a specific fine "tandem-cluster structure" and is synthesized through coordinate reactions of multiple isozymes of starch synthase (SS), starch branching enzyme (BE) and starch debranching enzyme (DBE). Recent studies involving mutants and transgenic plants have revealed distinct and overlapping functions of individual isozymes in starch biosynthesis, which can provide a biochemical basis for the variations in the fine structure and physicochemical properties of starch. In vivo studies strongly suggest that each isozyme in monocotyledonous plants contributes to the fine structure of amylopectin, playing a distinct role in the biosynthetic process in the endosperm. However, despite numerous studies to identify BE reaction products $^{1-3)}$, the mechanism of enzyme's action is poorly understood. To understand the regulation of starch biosynthesis, it is crucial to characterize the individual isozymes through a detailed in vitro analysis. In an in vitro analysis, enzymatic reactions can be conducted in the presence of only the target enzyme and the selected glucan substrate or primers with known structures without interference of other isozymes and classes of enzymes.

It is essential to resolve the fine structure of the reaction product to characterize the enzyme and reaction mechanism. For these purposes, the chain-length distribution of the BE or SS reaction product, which was debranched by using bacterial isoamylase and pullulanase, has been analyzed using fluorophore-assisted carbohydrate electrophoresis (FACE). ${ }^{4)}$ The method can be used to completely separate malto-oligosaccharides (MOS) with varying degree of polymerizations (DPs) ranging from 3 to approximately 100 as well as to determine the molar ratio of these MOS, because each chain is labeled with the fluorescent probe 8-amino1,3,6-pyrenetrisulfonic acid (APTS) at its reducing end.

Detailed analyses of debranched products from BE reactions using the FACE method revealed distinct chainlength preferences of individual BE isozymes. ${ }^{5}$ First, when $\mathrm{BE}$ was reacted with branched glucan, the minimum chainlengths of transferred chains and substrate chains were DP 6 and 12, respectively. In contrast, the minimum chain-length of linear glucan was DP 58 for BEI and DP > 100. Second, BEIIb nearly exclusively transferred chains of DP 7 and 6, while BEIIa formed a wide range of short chains from DP 6 to approximately 15 from outer chains of amylopectin and amylose. In contrast, BEI formed a variety of short chains and intermediate chains of $\mathrm{DP} \leq 40$ by attacking outer chains as well as inner chains of branched glucan, while BEIIa or BEIIb could only minimally or not at all attack inner chains, respectively. Comprehensive in vitro studies showed a functional distinction and an interaction of BE isozymes during amylopectin biosynthesis in cereal endosperm.

SSI was found to elongate short chains of DP $6^{-7}$ to primarily form chains of DP 8 and, to a lesser extent, chains of DP 9-10. This specific chain-length preference of SSI is in sharp contrast with that of SSIIa and SSIIIa since both enzymes can react to produce a wide range of chain-lengths, although it is probable that all SS isozymes recognize the free non-branched portion from the nonreducing end of the chain. Interestingly, the specific action of SSI may be that the $\alpha$-1,6-glucosidic linkages are localized in at least 2 different regions but not evenly located within the cluster of amylopectin.

In summary, in vitro studies can provide new insights into the mechanisms of regulation of starch biosynthesis. First, in vitro studies are useful for characterizing individual isozymes involved in starch biosynthesis, e.g., BE and SS. Second, through detailed analyses of enzymatic reaction products, reaction mechanisms of starch biosynthetic enzymes can be revealed. Third, plant species- and tissue-specific diversity exists in the composition and relative contribution of individual isozymes involved in starch synthesis.

\section{REFERENCES}

1) D. Borovsky, E.C. Smith and W.J. Whelan: On the mechanism of amylose branching by potato Q-enzyme. Eur. J. Biochem., 62, 307-312 (1976).

2 ) Y. Takeda, H.P. Guan and J. Preiss: Branching of amylose by the branching isoenzymes of maize endosperm. Carbohydr. Res., 240, 253-263 (1993).

3 ) H.P. Guan, P. Li, J. Imparl-Radosevich, J. Preiss and P. Keeling: Comparing the properties of Escherichia coli branching enzyme and maize branching enzyme. Arch. Biochem. Biophys., 342, 9298 (1997).

4 ) M.K. Morell, M.S. Samuel and M.G. O'Shea: Analysis of starch structure using fluorophore-assisted carbohydrate electrophoresis. Electrophoresis, 19, 2603-2611 (1998). 
5 ) Y. Nakamura, Y. Utsumi, T. Sawada, S. Aihara, C. Utsumi, M. Yoshida and S. Kitamura: Characterization of the reactions of starch branching enzymes from rice endosperm. Plant Cell Physiol., 51, 776-794 (2010).

*E-mail: nakayn@akita-pu.ac.jp $* * * * * * * * * *$

\section{Coordination of the Enzymes of Starch Biosynthesis}

\author{
Ian J. Tetlow, ${ }^{1, *}$ Fushan Liu, ${ }^{1}$ Elizabeth Lee, ${ }^{2}$ \\ Amina Makhmoudova, ${ }^{1}$ Nadya Romanova ${ }^{1}$ \\ and Michael J. Emes ${ }^{1}$ \\ ${ }^{1}$ Department of Molecular and Cellular Biology, \\ University of Guelph \\ (Ontario N1G 2W1, Canada) \\ ${ }^{2}$ Department of Plant Agriculture, University of Guelph \\ (Ontario N1G 2W1, Canada)
}

Starch granule architecture is defined by amylopectin, which is in turn made up of non-randomly branched glucan chains forming a semi-crystalline water-insoluble matrix. ${ }^{1)}$ The key enzyme classes involved in amylopectin biosynthesis are starch synthases (SS) responsible for glucan chain elongation via $\alpha-(1 \rightarrow 4)$-O-linkage formation, starch branching enzymes (SBE) which introduce groups of $\alpha-(1 \rightarrow 6)$ linked branch points to form clusters, and debranching enzymes which trim branch points, allowing the packing of large amounts of glucose within granules. Studies with amyloplasts from cereal endosperms indicate that specific SS and SBE isoforms physically associate with each other to form protein complexes in the plastid stroma and at the granule interface ${ }^{2-4}$. In particular, a trimeric assembly of SSI, SSIIa and SBEII has been identified in the endosperms of wheat and maize. ${ }^{2-3)}$ Analysis of this trimeric assembly with the hetero-bifunctional cross-linking reagent SULFOSBED suggests SSIIa is the central core of the protein complex. Assembly and disassembly of these protein complexes is dependent, respectively, on protein phosphorylation and dephosphorylation by plastidial protein kinases and protein phosphatases. Kinetic studies of SS/SBE-containing protein complexes suggest that SBEII in the complex has a greater affinity for glucan chains in amylopectin than the respective monomer. ${ }^{2)}$ It is proposed that SS/SBE protein complexes are involved in the formation of glucan clusters in amylopectin (which are made up of short- to intermediatesized glucan chains with clustered branch points), and eventually become entrapped within the matrix as starch granule-associated proteins (see below).

An increasing body of genetic and biochemical evidence supports the notion that starch granule-associated proteins are components of protein complexes involved in amylopectin synthesis. ${ }^{5-8)}$ For example, analysis of the amylose extender $\left(a e^{-}\right)$mutant of maize, which lacks the activity of the major form of SBE in the endosperm (SBEIIb), reveals novel protein complexes whose components are also partitioned in the starch granule as granule-associated proteins. ${ }^{8)}$ In $a e^{-}$mutants lacking expression of the SBEIIb protein (termed ae-1), SBEI replaces SBEIIb in the complex, which also contains starch phosphorylase and SBEIIa. ${ }^{8)}$ SBEI has a lower catalytic activity (i.e., frequency of branchpoint formation) and a higher affinity for branching long glucan chains than SBEII forms, ${ }^{9}$ and it is suggested that this complex is, at least in part, responsible for the observed 'high-amylose' phenotype (low branch frequency and long glucan chains in clusters) characteristic of $a e^{-}$starches. Another form of $a e^{-}$expresses a catalytically inactive form of SBEIIb (termed ae-2). Biochemical analysis of amyloplasts and starch from ae-2 endosperm indicates protein-protein interactions and starch physical properties which are distinct from those of wild type and ae-1. It is argued that the SS/ SBE protein complexes observed in cereal endosperm amyloplasts play an important role in glucan cluster formation of amylopectin, and impact the physicochemical properties of the resultant starches.

\section{REFERENCES}

1 ) S. Hizukuri: Polymodal distribution of the chain lengths of amylopectin, and its significance. Carbohydr. Res., 147, 342-347 (1986).

2 ) I.J. Tetlow, K.G. Beisel, S. Cameron, A. Makhmoudova, F. Liu, N.S. Bresolin, R. Wait, M.K. Morell and M.J. Emes: Analysis of protein complexes in amyloplasts reveals functional interactions among starch synthetic enzymes. Plant Physiol., 146, 1878-1891 (2008).

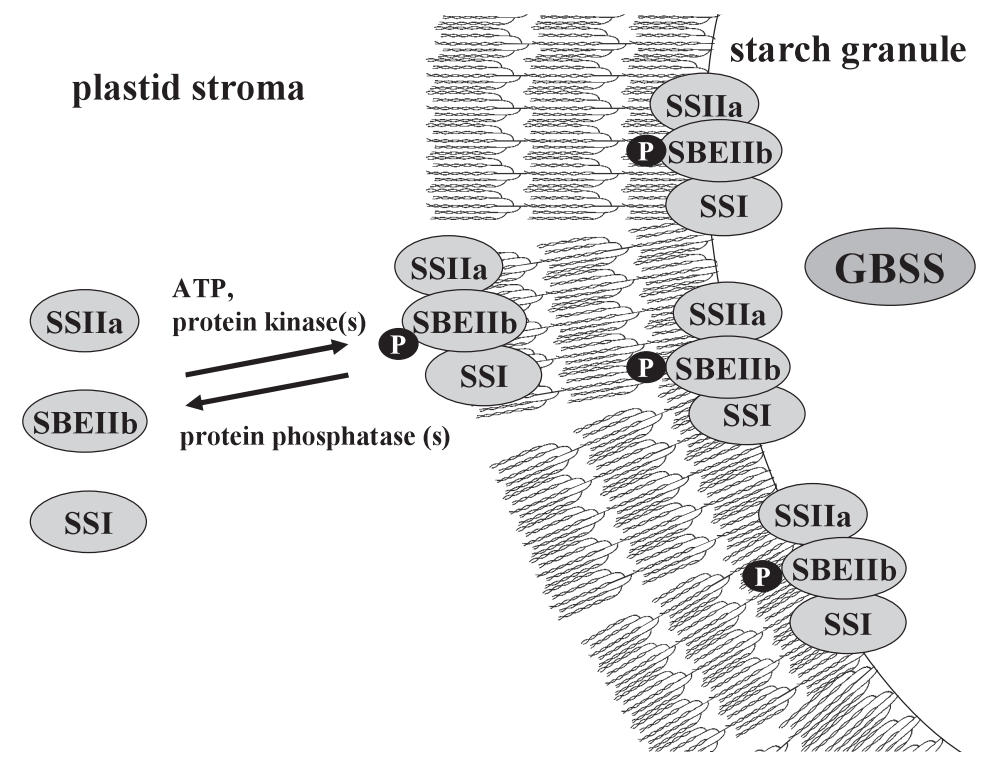


3 ) T.A. Hennen-Bierwagen, F. Liu, R.S. Marsh, S. Kim, Q. Gan, I.J. Tetlow, M.J. Emes, M.G. James and A.M. Myers: Starch biosynthetic enzymes from developing maize endosperm associate in multisubunit complexes. Plant Physiol., 146, 1892-1908 (2008).

4 ) T.A. Hennen-Bierwagen, Q. Lin, F. Grimaud, V. Planchot, P.L. Keeling, M.G. James and A.M. Myers: Proteins from multiple metabolic pathways associate with starch biosynthetic enzymes in high molecular weight complexes: A model for regulation of carbon allocation in maize amyloplasts. Plant Physiol., 149, 1541-1559 (2009).

5 ) M.K. Morell, B. Kosar-Hashemi, M. Cmiel, M.S. Samuel, P. Chandler, S. Rahman, A. Buléon, I.L. Batey and Z. Li: Barley sex6 mutants lack starch synthase IIa activity and contain a starch with novel properties. Plant J., 34, 173-185 (2003).

6 ) T. Umemoto and N. Aoki: Single-nucleotide polymorphisms in rice starch synthase IIa that alter starch gelatinization and starch association of the enzyme. Funct. Plant Biol., 32, 763-768 (2005).

7 ) B. Kosar-Hashemi, Z. Li, O. Larroque, A. Regina, M. Yamamori, M.K. Morell and S. Rahman: Multiple effects of the starch synthase II mutation in developing wheat endosperm. Funct. Plant Biol., 34, 431-438 (2007).

8 ) F. Liu, A. Makhmoudova, E.A. Lee, R. Wait, M.J. Emes and I.J. Tetlow: The amylose extender mutant of maize conditions novel protein-protein interactions between starch biosynthetic enzymes in amyloplasts. J. Exp. Bot., 60, 4423-4440 (2009).

9 ) T. Kuriki, D.C. Stewart and J. Preiss: Construction of chimeric enzymes out of maize starch branching enzymes I and II: activity and properties. J. Biol. Chem., 272, 28999-29004 (1997).

* E-mail: itetlow@uoguelph.ca $* * * * * * * * * *$

\section{Maize opaque 5 Encodes} Monogalactosyldiacylglycerol Synthase:

\section{Connections between Starch Biosynthesis and} Amyloplast Membrane Lipid Composition

\author{
Alan Myers ${ }^{1, *}$ \\ ${ }^{1}$ Department of Biochemistry, Biophysics, \\ and Molecular Biology, Iowa State University \\ (Ames, Iowa 50011, USA)
}

The maize opaque5 (o5) locus was shown to encode a protein highly similar to the monogalactosyldiacylglycerol (MGDG) synthase MGD1, which is necessary to produce the MGDG and subsequently the digalactosyldiacylglycerol (DGDG) that constitute $>90 \%$ of maize seedling lipid. Transition and insertion mutations of o5 that affect the vitreous nature of mature endosperm engendered an allelic series of lines with stepwise reduction in 05 function. These genotypes conditioned proportional reduction in 36:5 galactolipid abundance relative to $36: 6$ species, without affecting total galactolipid content. Effects of this membrane alteration on amyloplast and chloroplast function were characterized. Chloroplast development was altered such that protein complexes could not be normally incorporated into thylakoids. Endosperm development including starch production was altered, including changes in the frequency of glucan chains that form crystalline lamellae. Assembly of isoamylase-type starch debranching enzyme and an unidentified amylase was altered in mutant endosperm. The null allele caused kernel lethality owing to failure in both endosperm and embryo development. Starch assembly in null mutant endosperm was altered such that the regular arrangement of growth rings typical of virtually all normal granules was abolished. Thus, there is close relationship between amyloplast membrane structure and endosperm cell development and metabolism, including high structural order starch assembly. Low abundance galactolipids with five double bonds serve functions not replaced by the predominant 36:6 species, and MGD1 distinguishes the constituency of acyl groups on its diacylglycerol acceptor substrate.

*E-mail: ammyers@iastate.edu

\section{The Phosphorylation/Dephosphorylation Cycles in Transitory Starch Metabolism}

\author{
Martin Steup, ${ }^{1, *}$ Joerg Fettke ${ }^{2}$ and Mahdi Hejazi ${ }^{1}$ \\ ${ }^{1}$ Plant Physiology, Institute of Biochemistry and Biology, \\ University of Potsdam \\ (Karl-Liebknecht-Str. 24-25 Building 20, \\ 14469 Potsdam-Golm, Germany) \\ ${ }^{2}$ Mass Spectrometry of Biomolecules, \\ Institute of Biochemistry and Biology, \\ University of Potsdam \\ (Karl-Liebknecht-Str. 24-25 Building 20, \\ 14469 Potsdam-Golm, Germany)
}

Photosynthesis-competent eukaryots possess at least two distinct enzyme activities that mediate the phosphorylation of starch. Mutants that do not contain either of both enzyme activities exhibit a starch-excess phenotype and a reduced growth. Thus, phosphorylation appears to be an essential process within the starch metabolism and the development of the entire plant. Furthermore, the two mutant phenotypes clearly indicate that the two starch phosphorylating enzyme activities do not exert redundant functions.

Starch phosphorylation is mediated by two types of dikinases that utilize ATP as dual phosphate donor and transfer the terminal phosphate group to water (yielding orthophosphate) and the $\beta$-phosphate group first to a conserved histidine residue and, subsequently, to the $\alpha$-glucan to be phosphorylated. One of the two starch-related dikinases that has been designated as ATP: $\alpha$-1,4-glucan, water phosphotransferase (GWD; EC 2.7.9.4) phosphorylates glucosyl residues at the C6 position. ${ }^{1,2)}$ The second dikinase (designated as ATP: phospho- $\alpha-1,4$-glucan, water dikinase; PWD; EC 2.7.9.5) selectively transfers the $\beta$-phosphate group to C3 of glucosyl residues. ${ }^{3,4)}$ For the later enzyme, any starch-phosphorylating activity was detected only if native starch granules had been prephosphorylated with GWD and, therefore, it was assumed that this enzyme acts downstream of GWD.

For a more detailed analysis, native starch granules were replaced by a model system consisting of crystalline maltodextrins that represent either the highly ordered A or B allomorph. ${ }^{5,6)}$ The maltodextrins used in these studies comprise a relatively narrow range of DPs. Recoombinant GWD phosphorylates both allomorphs with far higher rates than any native starch granule preparation but solubilisation of the dextrins by heat treatment did abolish the phosphorylating activity. Thus, GWD appears to have a clear preference for acting on highly ordered insoluble dextrins. Phosphorylation is accompanied by a massive solubilisation of both neutral and phosphorylated maltodextrins. At a 
mechanistical level, these data explain the stimulatory effect that the phosphorylation of native starch granules exerts on the activity of hydrolytic enzymes, such as isoamylase and $\beta$-amylase. ${ }^{7)}$ Recombinant PWD phosphorylates crystalline maltodextrins provided they have been prephosphorylated with GWD and forms, to a significant extent, monophosphorylated $\alpha$-glucans. This implies that the dikinase is capable of acting on neutral maltodextrins. The prephosphorylation that is essential for the PWD activity appears to alter the physical order rather than chemical features of the $\alpha$-glucans.

Arabidopsis contains at least two plastidial phosphatases that contain a carbohydrate binding domain. Mutants lacking either of the phosphatases (or both) exhibit a starch-excess phenotype $^{8,9)}$ and, therefore, starch dephosphorylation appears to be equally important for the normal starch metabolism as the phosphorylating reactions. One of the two phosphatases, SEX4, dephosphorylates crystalline maltodextrins both in the C6- and C3-position. Dephosphorylation appears to counteract the phosphorylating activity of GWD and PWD as it results in a reordering of the $\alpha$-glucans. ${ }^{10)}$ The precise biochemical function of the second phosphatase remains to be elucidated.

\section{REFERENCES}

1 ) G. Ritte, J.R. Lloyd, N. Eckermann, A. Rottmann, J. Kossmann and M. Steup M: The starch-related R1 protein is an $\alpha$-glucan, water dikinase. Proc. Nat. Acad. Sci. USA, 99, 7166-7171 (2002).

2 ) G. Ritte, M. Heydenreich, S. Mahlow, S. Haebel, O. Kötting, M. Steup: Phosphorylation of C6- and C3-positions of glucosyl residues in starch catalysed by distinct dikinases. FEBS Lett., 580, 4872-4876 (2006).

3 ) L. Baunsgaard, H. Lütken, R. Mikkelsen, M.A. Glaring, T.T. Pham and A. Blennow: A novel isoform of glucan, water dikinase phosphorylates prephosphorylated $\alpha$-glucans and is involved in starch degradation in Arabidopsis. Plant J., 41, 595-605 (2005).

4 ) O. Kötting, K. Pusch, A. Tiessen, P. Geigenberger, M. Steup and G. Ritte Identification of a novel enzyme required for starch metabolism in Arabidopsis leaves. The phosphoglucan, water dikinase. Plant Physiol., 137, 242-252 (2005).

5 ) M. Hejazi, J. Fettke, O. Paris and M. Steup: Glucan, water dikinase phosphorylates crystalline maltodextrins and thereby initiates solubilisation. Plant J., 55, 323-334 (2008).

6 ) M. Hejazi, J. Fettke, O. Paris and M. Steup: The two plastidial starch-related dikinases sequentially phosphorylate glucosyl residues at the surface of both the A- and the B-type allomorphs of crystallized maltodextrins but the mode of action differs. Plant Physiol., 149, 1541-1559 (2009).

7 ) C. Edner, J. Li, T. Albrecht, S. Mahlow, M. Hejazi et al.: Glucan, water dikinase activity stimulates breakdown of starch granules by plastidial $\beta$-amylases. Plant Physiol., 145, 17-28 (2007).

8 ) O. Kötting, D. Santelia, C. Edner, S. Eicke, T. Marthaler, et al.: SEX4, a glucan phosphatase, dephosphorylates amylopectin at the surface during starch breakdown in Arabidopsis leaves. Plant Cell, 21, 334-346 (2009).

9 ) S. Comparat-Moss, O. Kötting, M. Stellter, C. Edner, A. Graf et al: A putative phosphatase, LSF1, is required for normal starch turnover in Arabidopsis leaves. Plant Physiol., 152, 685-698 (2010).

10) M. Hejazi, J. Fettke, O. Kötting, S.C. Zeeman and M. Steup: The laforin-like dual-specificity phosphatase SEX4 from Arabidopsis thaliana hydrolyses both C6- and C3-phosphate esters introduced by starch-related dikinases and thereby affects phase transition of a-glucans. Plant Physiol., 152, 711-722 (2010).

*E-mail:msteup@uni-potsdam.de

\section{Abstracts for Closed Symposium}

\section{Septum Formation in Amyloplasts Produces Compound Granules in the Rice Endosperm}

\author{
Min-Soo Yun ${ }^{1}$ and Yasushi Kawagoe ${ }^{1, *}$ \\ ${ }^{1}$ Division of Plant Sciences, National Institute of \\ Agrobiological Sciences \\ (2-1-2 Kannondai, Tsukuba 305-8602, Japan)
}

The plastid division apparatus evolved primarily from the cell division apparatus of the cyanobacterial progenitor of chloroplasts and consists of protein complexes on the stromal side of the inner envelope membrane (IEM) and on the cytosolic side of the outer envelope membrane (OEM). Unlike binary fission of chloroplasts in the leaves of rice (Oryza sativa) and Arabidopsis (Arabidopsis thalinana), amyloplasts in the rice endosperm divide simultaneously at multiple sites. ${ }^{1}$ The apparently different modes of division between chloroplast and amyloplasts suggest that plants have acquired multiple plastid division mechanisms. In the rice endosperm, each amyloplast produces compound granules consisting of several dozen polyhedral, sharpedged, and easily separable granules, whereas in other cereals, including wheat (Triticum aestivum), barley (Hordeum vulgare) and maize (Zea mays), each amyloplast synthesizes 1 granule. Despite extensive studies of starch synthesis mutants in cereals, the molecular mechanisms involved in compound granule synthesis in rice have remained elusive. We expressed green fluorescent protein fused to rice Brittle1 (BT1), an IEM protein, to determine whether IEM divides granules inside the amyloplast. Confocal microscopy revealed that a septum-like structure, or cross wall, containing BT1-GFP divides granules in the amyloplast. ${ }^{2)}$ Loss-of-function and overexpression analyses have suggested that plastid division proteins, including FtsZ, Min, and PDV2, play critical roles in amyloplast division as well as in the synthesis of the septum-like structure between granules in the amyloplast. We propose that successive septum syntheses, which create sections inside the amyloplast, and de novo granule synthesis in each section are primarily responsible for compound granule synthesis, and that in amyloplast division, septum synthesis is accompanied by OEM constriction; septum synthesis without OEM constriction leads to synthesis of characteristic compound granules.

\section{REFERENCES}

1 ) M.-S. Yun and Y. Kawagoe: Amyloplast division progresses simultaneously at multiple sites in the endosperm of rice. Plant Cell Physiol., 50, 1617-1626 (2009).

2 ) M.-S. Yun and Y. Kawagoe: Septum formation in amyloplasts produces compound granules in the rice endosperm and is regulated by plastid division proteins. Plant Cell Physiol., 51, 14691479 (2010).

* E-mail: kawagoe@nias.affrc.go.jp

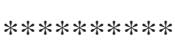




\section{Function of Starch Synthase (SS) Isozymes in Rice Endosperm}

\author{
Naoko Fujita, ${ }^{1, *}$ Hiroki Asai, ${ }^{1}$ Rui Satoh, ${ }^{1}$ Aki Hayashi, ${ }^{1}$ \\ Momoko Kodama, ${ }^{1}$ Rumiko Itoh, ${ }^{1}$ Satomi Aihara ${ }^{1}$ and \\ Yasunori Nakamura ${ }^{1}$ \\ ${ }^{1}$ Faculty of Bioresource Science, \\ Akita Prefectural University \\ (241-438 Kaidobata-nishi, Shimoshinjo-nakano, \\ Akita 010-0195, Japan)
}

Starch is one of the most important carbohydrates for sustaining life on earth. It consists of 2 homopolymers of the $\alpha$-D-glucosyl unit, amylose and amylopectin. At least 4 classes of enzymes catalyze the reactions of starch biosynthesis: ADP-glucose pyrophosphorylase (AGPase), starch synthase (SS), starch branching enzyme (BE) and starch debranching enzyme (DBE). Moreover, AGPase has 6 subunits, $3 \mathrm{BE}, 11 \mathrm{SS}$ and $4 \mathrm{DBE}$ isozymes in rice. Multiple isozymes mediate starch biosynthesis in green plants such as rice, indicating that these organisms have evolved the capacity to synthesize highly organized amylopectins through the functional specificity of different isozymes for each tissue, developmental stage and different substrates. Elucidating the function of each isozyme is therefore essential for understanding of the advanced starch biosynthesis mechanisms characteristic of higher plants.

Many starch biosynthesis-related mutant lines have been isolated in rice. The wild-type japonica rice shows a translucent seed morphology. In contrast, some mutants have specific seed morphology. For example, an SSI-deficient mutant seed was nearly the same as the wild type seed and an SSIIIa-deficient mutant had a white core, whereas a double mutant of both SS showed an opaque morphology.

Rice varieties cultivated worldwide are classified into 2 groups, indica-types and japonica-types, each possessing distinct qualities that greatly influence rice cooking and processing methods for food applications. Most of these properties, if not all, are regulated by 2 starch synthase genes, namely GBSSI and SSIIa. In typical indica rice cultivars, GBSSI and SSIIa are active, whereas in typical japonica cultivars, the activity of these isozymes is reduced to approximately $10 \%$ due to mutations in both genes. These mutations result in a sticky texture and low gelatinization temperature of japonica rice starch derived from its low amylose content, as well as S-type amylopectin, respectively. Japonica rice cultivars are further classified into non-glutinous and glutinous cultivars. The latter is a null gbss1 mutant and accumulates amylose-free starch in the endosperm.

Starch synthase elongates $\alpha$-glucans by adding glucose residues from ADP-glucose. Rice 11 SS isozymes can be further separated into 6 types. GBSS isozymes are related to amylose and extra-long chains of amylopectin biosynthesis, whereas the other SSs are related to amylopectin biosynthesis. SSI and SSIIIa account for approximately 60 and 30\% of total soluble SS activity, respectively, in the developing rice endosperm.

To determine the function of these SS isozymes, we isolated ss1 and ss3a mutants by reverse genetics using a retrotransposon Tos17 insertion mutant stock. SSI, SSIIa and SSIIIa are the most important SS isozymes related to the amylopectin biosynthesis in the rice endosperm. Differences in chain-length distribution of these SS isozyme mutants revealed a distinct role of chain elongation in amylopectin. SSI specifically elongates a narrow range of short chains with DP 6-7 and adds a limited number of glucose residues (DP 2-4), while SSIIa and SSIIIa elongate chains with DP $\leq$ 12 and long $B$ chains to $12 \leq \mathrm{DP} \leq 24$ and longer B chains, respectively. Importantly, the functions of individual SS isozymes appear to overlap with each other. In summary, in wild type japonica rice, branch chains with DP 6 or 7 produced by BEIIb are initially elongated by SSI. Next, chains elongated by SSI should be further elongated by SSIIa to form longer chains typical of indica rice. Long chains that connect into clusters were elongated by SSIIIa.

Currently, we are attempting to produce double recessive rice lines mutated in their starch biosynthesis-related genes. Many studies involving double starch mutants of maize were conducted in the 1980's. At that time, few starch mutant genes were identified; however, now, we understand the relationships between mutants and related genes. We can understand the relationships between the characteristics of starch and starch biosynthesis-related isozyme genes. This information provides insights for the production of tailormade starch for accumulation in storage tissues. The function of minor starch biosynthesis-related isozymes is typically masked in wild-type by increasing the number of isozymes developed by higher plants as a compensatory system. To understand the functions of these isozymes that have not been characterized, activities of other functionally overlapping isozymes must be eliminated. However, we understand that double recessive japonica rice mutants can be easily sterilized due to a leaky $\triangle S S I I a / \triangle G B S S I$.

To obtain the double mutant of major SS isozymes, an ss1 null mutant was crossed with an ss3a null mutant. Opaque seeds, in which the phenotype was different from that in parent lines, appeared in the $F_{2}$ segregation. $F_{3}$ seeds of the self-pollinated seeds of $F_{2}$ opaque seeds were segregated again, indicating that opaque seeds are not double recessive homozygous. Moreover, 2 types of $\mathrm{F}_{3}$ segregations appeared, a TO line was separated into translucent and opaque seeds, and a WO line was separated into white core and opaque seeds. To determine the genotype of TO and WO opaque seeds, seeds were germinated and DNA was extracted from young leaves for PCR selection. Opaque seeds of TO and WO lines showed an ss1ss1/SS3ass3a and SS1ss1/ss3ass3a genotype, respectively, whereas translucent seeds of the TO line and white core of the WO line had the same genotype as their parent mutants, $\triangle S S I$ and $\triangle S S I I I a$, respectively. Phenotypic analyses revealed lower SS activity in the endosperm of these lines than in that of the parent mutant lines; this is because these seeds have a different number of copies of SSI and SSIIIa genes in the heterozygous state. When either of these genes was heterozygous, dry seeds showed an opaque morphology, unique amylopectin structure, high amylose content, large proportion of round shape starch granules, and low viscosity of gelatinized starch. The high amylose content may be derived from pleiotropic effects, primarily the high amount of GBSSI protein and high AGPase activities.

Importantly, both types of opaque seeds maintained more than $80 \%$ of seed weight compared to wild-types, indicating 
that reduction or deficiency of SSI and SSIIIa results in complementation of chain elongation by other SSs, or optimization of the remaining SS activity by environmental conditions. However, we failed to identify any developing endosperms that had completely lost SSI and SSIIIa activity in both lines, indicating that homozygous lines of double recessive SSI and SSIIIa genes must be sterile. These results suggest that either SSI or SSIIIa activity is necessary for starch biosynthesis in the endosperm, and no other SS can complement this activity.

To obtain a double recessive homozygous mutant, an ss1 leaky mutant had been isolated and crossed with an ss $3 a$ mutant. A non-segregated opaque line that had retained a low level of SSI activity was successfully obtained from a selection of double recessive homozygous lines using PCR in $F_{2}$ opaque seeds. The use of leaky mutants to prevent sterility, therefore, proved to be a useful method.

Acknowledgements: This work was partially supported by the Program for the Promotion of Basic and Applied Research for Innovations in Bio-oriented Industry and a Grant-in-Aid for Scientific Research (B) (19380007).

*E-mail: naokof@akita-pu.ac.jp

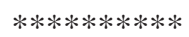

\section{Saturation Mutant Library Derived from $N$-methyl- $N$-nitrosourea Treatment of Fertilized Egg Cells in Rice}

\author{
Hikaru Satoh, ${ }^{1, *}$ Aiko Nishi ${ }^{1}$ and Tetsuhiro Nakamura ${ }^{1}$ \\ ${ }^{1}$ Institute of Genetic Resources, Faculty of Agriculture, \\ Kyushu University \\ (6-10-1 Hakozaki, Higashi-ku, Fukuoka 812-8581, Japan)
}

Storage starch and transient starch are long-term and short-term energy storages in plants, respectively. Recent studies reveal that the genes for metabolic pathways involved in the synthesis and degradation of starch are different between sink and source tissues. However, the details of the genetic regulation of storage and transient starch metabolisms and the interrelationship between them are yet remained to be elucidated. In addition, although the outline of starch biosynthesis has been proposed, many mechanistic questions such as initiation of starch biosynthesis, biosynthesis of starch granule, biogenesis of amyloplast, metabolic regulation of energy supply and substrate metabolism, are remained to be solved.

Rice (Oryza sativa L.) is one of the most suitable species for functional genomics of carbohydrate metabolism in plants because of the availability of the full genome sequence and the easy handling of both storage and synthetic tissues. Mutants are helpful materials to understand the function of genes and proteins, and the metabolic regulation system of starch biosynthesis. Identification of genetic mutations will reveal new insights on these processes. Mutations can be classified into the insertional mutation and the point mutation such as nucleotide (s) deletion and single nucleotide substitution mutations, and there are three types of mutations, nonsense (knockout or null), leaky (knockdown) and missense (non-synonymous; nucleotide substitution resulting in amino acid replacement) in the point mutation. In particular, missense mutations will play an important role in functional genomics, not only elucidating the functions of individual genes but also contributing fundamental information in proteomics, metabolomics and phenomics, so called OMICS science, because they can produce weak, moderate, or severe modifications of protein function, depending on the position and type of amino acid replaced. This characteristic of missence mutations can be very helpful for determining the role of individual amino acids on the function of a given protein.

Artificially induced mutations are expected to play an important role in generating bioresources for post-genome science in plants as well as in vertebrates and microbes; the power of such mutations is that they enable comparison of the mutant and corresponding wild-type genes in a genetically uniform background. Of the many kinds of chemical mutagens that have been employed to induce nucleotide substitution mutations, $N$-methyl- $N$-nitrosourea (MNU) have been reported to be one of the most potent mutagens inducing mutations in vertebrate and microbial cells. The mutagenic effect of MNU is characterized by specific nucleotide substitution, in particular, predominantly GC to AT transitions, since O6-methylguanine, induced in DNA by MNU treatment, gave rise to $\mathrm{G}: \mathrm{C} \rightarrow \mathrm{A}: \mathrm{T}$ transition mutations via mispairing with thymine during DNA replication., ${ }^{1,2}$ Another unique characteristic of MNU mutagenesis is that its action is tightly associated with DNA replication. It was demonstrated that, in a synchronized E. coli culture, MNU-induced mutations did not occur at random throughout the replicating chromosome but were preferentially localized in the region of the replicating fork, $\left.{ }^{3,4}\right)$ suggesting that MNU mutates predominantly genes under the replication of DNA.

On the development of mutant library in post genome science, two important subjects for mutation induction are to increase the mutation frequency to cover all of genes in genome and to control the mutation spectrum. A high mutation frequency could be expected by the treatment of zygotes at the single-cell stage because it would reduce or eliminate the possibility of chimera formation and diplontic selection (loss of mutated cells due to competition between mutated and non-mutated cells within the plant). From these reasons, we treated the fertilized egg cells at the single-cell stage after flowering in the japonica rice cultivars Kinmaze and Taichung 65 (T65) with the chemical mutagen MNU. At dusk, 1 to 2 days before treatment, rice plants at the maximum flowering stage were transplanted from the paddy field to plastic pots, and the pollinated spikelets were cut off with scissors. To synchronize the developmental stage of the fertilized egg cells as much as possible, the spikelets that opened for $1 \mathrm{~h}$ from 11:30 to 12:30 were kept for the MNU treatment and the unopened spikelets were cut off. The panicles were soaked in MNU solution of $1.0 \mathrm{mM}$ for $1 \mathrm{~h}$ at around $24^{\circ} \mathrm{C}$ in the dark to avoid the decomposition by light including UV ray. The panicles were rinsed several times immediately after treatment and then washed for more than $24 \mathrm{~h}$ in running tap water under open field as sunlight in day time is expected to accelerate the decay of MNU. he efficiency of MNU treatment of fertilized egg cells at the single-cell stage in rice was about twice that of dry seeds, ${ }^{5)}$ perhaps due to the absence of diplontic selection and chimera formation, and the multiple replicating forks of DNA when the actively 
living cells under replication of DNA were mutagenized, instead of the embryos with multicellular and dormant cells.

TILLING (Targeting Induced Local Lesions IN Genomes) method is one of the most excellent methods to identify the precise mutation frequency per gene loci in the mutation treatment. ${ }^{6)}$ A modified TILLING system using non-labeled primers and fast capillary gel electrophoresis was applied to high-throughput detection of single-nucleotide substitution mutations in $\mathrm{M}_{2}$ plants derived from MNU treatment of fertilized egg cells at the single-cell stage. ${ }^{7)}$ When the mutation for three gene loci selected randomly were analyzed in the MNU treated population, the mutation rate of an $\mathrm{M}_{2}$ mutant population was calculated as $7.4 \times 10^{-6}$ per nucleotide, which represents one mutation in every $135-\mathrm{kb}$ genome sequence. At this frequency, one can expect 7.4 single nucleotide substitution mutations in every $1 \mathrm{~kb}$ of genome sequence when using 1,000 $\mathrm{M}_{2}$ mutant lines. The results of TILLING analysis strongly suggest that this mutant library covers all of the loci in the rice genome; in other words, it appears to represent a saturation mutant library with multiple allelic mutants in rice. The mutations were very evenly distributed over the regions examined. In addition, 22 of the 24 nucleotide substitution mutations analyzed for three gene loci (92\%) were transitions of $\mathrm{G}$ to A or $\mathrm{C}$ to T. ${ }^{7)}$ Substitutions from $\mathrm{G}$ to $\mathrm{A}$ and $\mathrm{C}$ to $\mathrm{T}$ were 50 and $42 \%$, respectively, as expected from alkylation of the guanine residue by MNU and the remaining two were transversions from AT to TA. Similar results were obtained for mutagenesis of other genes, ${ }^{8,9)}$ providing further evidence that MNU induces predominantly GC to AT transitions in plants, as well as in microbes and vertebrates. Additional property of MNU mutagenesis is characterized by the high frequency of nonsense mutations derived from the mutations of consensus sequence at the splicing site of intron/exson, ${ }^{10)}$ which result the loss of splicing and, in turn, lead to formation of a stop codon by a frame shift in the sequence, ${ }^{8,9)}$ in addition to from the formation of stop codons. ${ }^{11)}$

Mutant populations are indispensable genetic resources for functional genomics in all organisms. We have developed more than two thousand mutant lines modifying not only storage starch ${ }^{12)}$ but also transient starch and maintained at Kyushu University. In addition, we have cataloged 6,000 mutant lines derived from the MNU treatment of fertilized egg cell in two japonica c.vs, "T 65" and "Kinmaze" in rice. ${ }^{13)}$ Mutant seeds can be obtained by requesting them at Oryzabase (http://www.shigen.nig.ac.jp/rice/oryzabase/top/ top.jsp). The results of TILLING analysis strongly suggest that this mutant library covers all of the loci in the rice genome; in other words, it appears to represent a saturation mutant library with multiple allelic mutants in rice. This library of MNU-induced mutants will be a fundamental functional genomics resource in the identification of mutations in any rice gene and, hence, will complement other available mutant gene resources.

Acknowledgements: This research was partly supported by the Japan Society for the Promotion of Science, by the Bio-oriented Technology Research Advancement Institution and by the National Bioresource Project, Japan.

\section{REFERENCES}

1 ) J. Engelbergs, J. Thomale and M.F. Rajewsky: Role of DNA repair in carcinogen-induced ras mutation. Mutation Res., 450, 139-153 (2000).

2 ) M.S. Cooke, M.D. Evans, M. Dizdaroglu and J. Lunec: Oxidative DNA damage: mechanisms, mutation, and disease. FESEB J., 17, 1195-1214 (2003).

3 ) T.A. Hince and S. Neale: Effect of Escherichia coli growth rate on the number of mutations induced by $N$-methyl- $N$-nitrosourea. Proc. Soc. Gen. Microbiol., 2, 53 (1975).

4 ) T.A. Hince and S. Neale: A comparison of the mutagenic action of the methyl and ethyl derivatives of nitrosamides and nitrosamidines on Escherichia coli. Mutation Res., 24, 383-387 (1974).

5 ) H. Satoh and T. Omura: Induction of mutation by the treatment of fertilized egg cell with $N$-methyl- $N$-nitrosourea in rice. J. Fac. Agric. Kyushu Univ., 24, 165-174 (1979).

6 ) B.J. Till, S.H. Reynolds, E.A. Greene, C.A. Codomo, L.C. Enns, J.E. Johnson, C. Burtner, A.R. Odden, K. Young, N.E. Taylor et al.: Large-scale discovery of induced point mutations with highthroughput TILLING. Genome Res., 13, 524-530 (2003).

7 ) T. Suzuki, M. Eiguchi, T. Kumamaru, H. Satoh, H. Matsusaka, K. Moriguchi, Y. Nagato and N. Kurata: MNU-induced mutant pools and high performance TILLING enable finding of any gene mutation in rice. Mol. Genet. Genomics, 279, 213-223 (2008).

8 ) H. Satoh, K. Shibahara, K. Tokunaga, A. Nishi, M. Tasaki, S. K. Hwang, T.W. Okita, N. Kaneko, N. Fujita, M. Yoshida et al.: Mutation of the plastidic $\alpha$-glucanphosphorylase gene in rice affects the synthesis and structure of starch in the endosperm. Plant Cell, 20, 1833-1849 (2008).

9 ) K.C. She, H. Kusano, K. Koizumi, H. Yamakawa, M. Hakata, T. Imamura, M. Fukuda, N. Naito, Y. Tsurumaki, M. Yaeshima et al.: A novel factor FLOURY ENDOSPERM 2 is involved in regulation of rice grain size and starch quality. Plant Cell, 22, 3280-3294 (2010).

10) J.W.S. Brown: Arabidopsis intron mutations and pre-mRNA splicing. Plant J., 10, 771-780 (1996).

11) L.E. Maquat: Nonsense-mediated mRNA decay: Splicing, translation and mRNA dynamics. Nat. Rev. Mol. Cell Biol., 5, 89-99 (2004).

12) H. Satoh, A. Nishi, N. Fujita, A. Kubo, Y. Nakamura, T. Kawasaki and T.W. Okita: Isolation and characterization of starch mutants in rice. J. Appl. Glycosci., 50, 225-230 (2003).

13) H. Satoh, H. Matsusaka and T. Kumamaru: Use of $N$-methyl- $N$ nitrosourea treatment of fertilized egg cells for saturation mutagenesis of rice. Breed. Sci., 60, 475-485 (2010).

* E-mail: hsatoh@agr.kyushu-u.ac.jp

$* * * * * * * * * *$

\section{Relationship between Structure and Physicochemical Properties of Rice Starches}

\author{
Naoyoshi Inouchi, ${ }^{1, *}$ Tetsuya Horibata, ${ }^{1}$ Hideo Hibiu, \\ Yoshiko Nakaura ${ }^{1}$ and Tomio Itani ${ }^{2}$ \\ ${ }^{1}$ Faculty of Life Science and Technology, \\ Fukuyama University \\ (1 Sanzo, Gakuen-cho, Fukuyama, \\ Hiroshima 729-0292, Japan) \\ ${ }^{2}$ Faculty of Life and Environmental Sciences, \\ Prefectural University of Hiroshima \\ (562 Nanatsuka-cho, Shobara, \\ Hiroshima 727-0023, Japan)
}

\section{Relationship between structure and physicochemical properties of endosperm starches of rice originated in Japan and other countries.}

Properties of rice starches were investigated using 4 types of endosperm starches, including waxy, low amylose (AM), medium AM and high AM starches from rice cultivars bred 


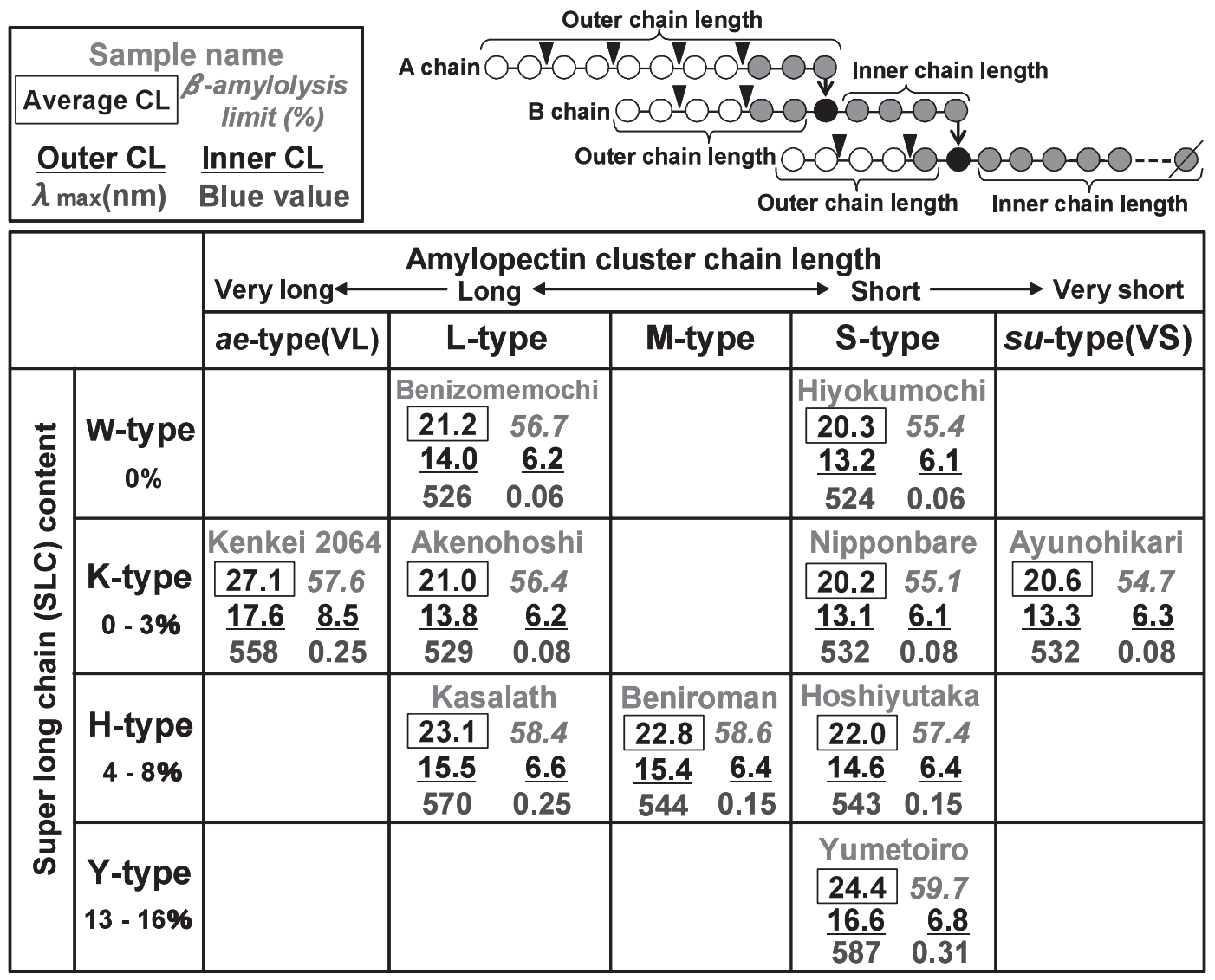

Fig. 1. Structural characteristics of waxy rice starches and APs purified from non-waxy rice starches.

in Japan and other countries. Rice starches were prepared from polished rice using a cold alkali method. There was a highly negative relationship between real AM content and peak viscosity measured using a Rapid Visco Analyzer (RVA) from non-waxy starches. In high AM starches, there were 2 types in Japan and various types in other countries of rice starch samples with similar apparent AM contents, different contents of super-long chains (SLC) in amylopectin (AP), and different values in peak viscosity and setback of starches measured by RVA. Since there was a highly positive relationship between SLC contents and setback, SLC in AP appears to have a significant influence on starch setback. Both the side chain length distributions within the short chain range of AP and the SLC content of AP significantly influence the gelatinization temperature of rice starches. The SLC in AP contributed to neither swelling nor dissolution of rice starches in water at $75^{\circ} \mathrm{C}$. Starches of non-waxy cultivars of indica and Chinese indica contained decreased amounts of branch chains with a degree of polymerization (DP) of 6-12 (Fr. A). The Fr. A content correlated negatively with the peak temperature of gelatinization of rice starches measured by DSC. There was a high positive relationship between the amounts of Waxy (Wx) protein and SLC contents in starch. This demonstrates that the Wx protein is involved in SLC synthesis. Finally, we propose a scheme for the relationship between SLC and Fr. A contents of AP as a classification of endosperm starches in rice.

2. Fine structure of rice amylopectins with different chainlength distributions and SLC contents.

Structural characteristics were investigated for waxy rice starches and the APs purified from non-waxy rice starches using Schoch's method modified by Takeda et al. with different chain length (CL) distributions and SLC contents. Figure 1 shows the average CL, $\beta$-amylolysis limit, average outer CL, average inner CL, $\lambda$ max (wavelength at absorption maximum) and blue value ( $\left.A_{680}\right)$ of iodine complexes of the rice APs. Average CL and outer CL were highest in the very long type of AP cluster CL. The highest $\beta$-amylolysis limit, $\lambda_{\max }$, and blue value were observed in APs with high SLC contents. The average inner CL of AP was 6-7 and was similar among APs, except for the very long type of AP cluster CL.

$$
\begin{aligned}
& * \text { E-mail: inouchi@fubac.fukuyama-u.ac.jp } \\
& * * * * * * * * * *
\end{aligned}
$$

\section{Analysis of Starch Biosynthesis in Novel Sweetpotatoes with Starches Pasting at Low Temperature}

Yasuhiro Takahata, ${ }^{1, *}$ Masaru Tanaka, ${ }^{1}$ Kenji Katayama, ${ }^{1}$ Motoyasu Otani, ${ }^{2}$ Kanefumi Kitahara, ${ }^{3}$ Takayuki Umemoto, ${ }^{4}$ Osamu Nakayachi, ${ }^{2}$ Hiroki Nakayama ${ }^{1}$ and Masaru Yoshinaga ${ }^{1}$ ${ }^{1}$ National Agricultural Research Center for Kyushu Okinawa Region (KONARC), NARO

(6651-2 Yokoichi, Miyakonojo 885-0091, Japan)

${ }^{2}$ Research Institute for Bioresources and Biotechnology, Ishikawa Prefectural University (Nonoichi, Ishikawa 921-8836, Japan)

${ }^{3}$ Faculty of Agriculture, Kagoshima University (1-21-24 Korimoto, Kagoshima 890-0065, Japan)

${ }^{4}$ National Institute of Crop Science (NICS), NARO

(Tsukuba, Ibaraki 305-8518, Japan) 
Sweetpotatoes are used in Japan for starch production as well as for table use, various kinds of snack foods, confectioneries, traditional foods and alcohol-containing beverages. The qualities and characteristics of starch are very important for sweetpotato utilization, but the qualitative diversity of sweetpotato starch is very narrow. Distinct differences in the starch properties of the sweetpotato have not been reported. Recently, a new cultivar, Quick Sweet (QS), was released; starch from QS features a low pasting temperature, ${ }^{1,2)}$ although the biochemical background of this property is unknown. Changes in amylopectin short chain distribution point to the critical role of starch synthases in QS. However, there have been few studies on starch synthase in sweetpotatoes, ${ }^{3,4)}$ except for a study showing granulebound starch synthase (GBSS) affecting amylose synthesis. $^{5-10)}$ In this report, we summarize our analysis of starch synthase isoform II (SSII) in sweetpotato storage roots and the effect of reduced SSII expression on starch properties in transgenic sweetpotatoes.

SSII expression in the storage roots of QS with low pasting temperature starch was notably lower than in cultivars with normal starch. Moreover, nucleotide sequence analysis suggests that most SSII transcripts in the cultivar with low pasting temperature starch were inactive alleles. ${ }^{11)}$ Western blot analysis using an antibody against pea embryo SSII

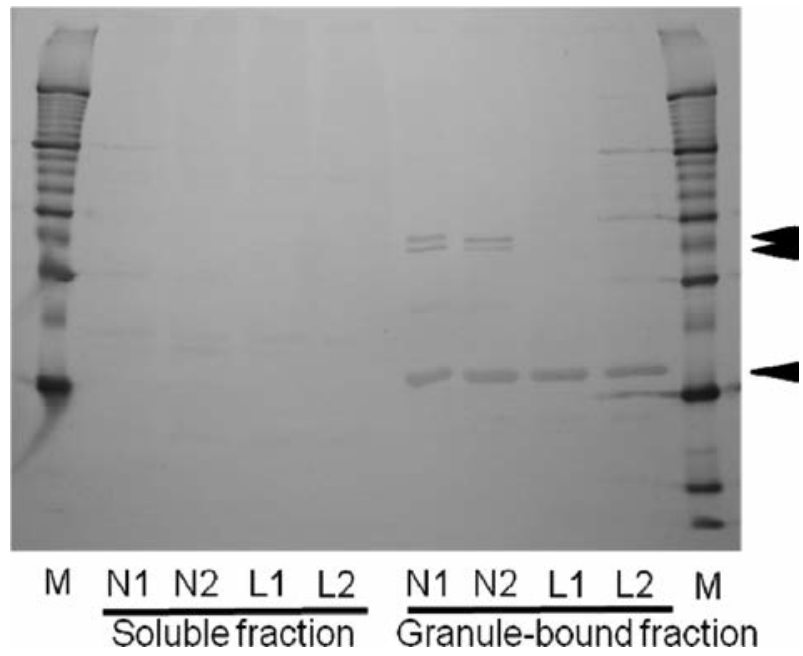

Fig. 1. Western blot analysis using an antibody against pea embryo SSII.

N1, N2: normal; L1, L2: low temperature pasting; M: molecular marker. The upper 2 arrows indicate protein bands only detected in N1 and N2. The lowest arrow presumably indicates GBSS. revealed a difference in the granule-bound fraction (Fig. 1). In the granule-bound fraction, reactions were detected only in the normal type (upper 2 arrowheads), whereas no reaction was detected in the soluble fraction. We also detected another reaction in the granule-bound fraction of both normal and low temperature pasting types (the lowest arrowhead), which is thought to represent a reaction leading to GBSS production. Sodium dodecyl sulfate-polyacrylamide gel electrophoresis (SDS-PAGE) of the granule-bound protein fraction indicated a difference between the low temperature pasting type and the normal type (Fig. 2). Some protein bands were not present in samples from QS. To investigate which proteins were eliminated, the 2 corresponding protein bands detected in the normal type were identified using mass spectrometry analysis. The results derived from the 2 bands were very similar. Four or five peptide fragments were matched, and the bands were found to contain sweetpotato starch synthase II. Mass spectrometry results also indicated the presence of sweetpotato starch branching enzyme (SBE) II in both bands. SBE II in sweetpotato reportedly decreases DP by $6-10 .{ }^{10)}$ In the low temperature pasting type, although the bands deduced as protein complexes of SSII and SBEII were eliminated, the profile of short chain distribution of amylopectin $^{1)}$ did not show SBEII suppression. The presence of SBEII in the granule-bound fraction may be due to the presence of protein complexes with SSII as secondary effect of starch biosynthesis. Trimeric assembly of SSI, SSIIa and SBEII in the endosperms of wheat and maize has been reported. ${ }^{12,13)}$ The SSI of sweetpotato, which is still unidenti-

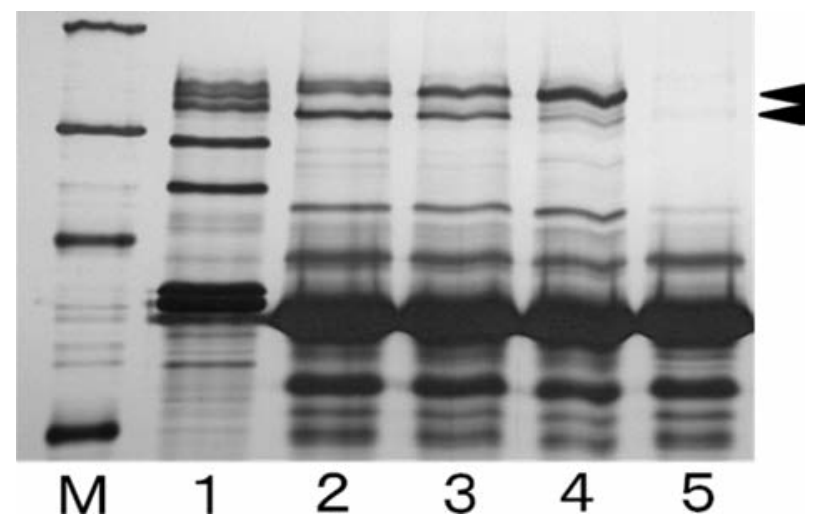

Fig. 2. SDS-PAGE analysis of granule-bound fraction protein.

1, wheat (Norin61); 2-4, normal; 5, low temperature pasting; $\mathrm{M}$, molecular marker. The upper 2 arrows indicate protein bands provided for mass spectrometry analysis (Lane 3, Beniazuma).

Table 1. Physiological trait and RVA starch properties of transgenic sweetpotato plants (cv. White Star).

\begin{tabular}{|c|c|c|c|c|c|c|c|c|}
\hline Plant & $\begin{array}{c}\text { Weigt of } \\
\text { aerial part }^{\mathrm{a})}\end{array}$ & $\begin{array}{l}\text { Storage root } \\
\text { weight }^{\mathrm{a})}\end{array}$ & $\begin{array}{c}\text { Dry matter } \\
\text { content }^{\mathrm{b}, \mathrm{c})}\end{array}$ & $\begin{array}{c}\text { Starch } \\
\text { content }^{\mathrm{b})}\end{array}$ & $\begin{array}{c}\text { Pasting } \\
\text { temp. }\left({ }^{\circ} \mathrm{C}\right)^{\mathrm{d})}\end{array}$ & $\begin{array}{c}\text { Peak } \\
\text { visc. }(\text { RVU) })^{\mathrm{d})}\end{array}$ & $\begin{array}{l}\text { Breakdown } \\
(\mathrm{RVU})^{\mathrm{d})}\end{array}$ & $\begin{array}{l}\text { Setback } \\
(\mathrm{RVU})^{\mathrm{d})}\end{array}$ \\
\hline White star & 72.0 & 101.3 & 29.1 & 21.1 & 75.3 & 185 & 80 & 130 \\
\hline White star & 75.1 & 89.1 & 27.5 & 19.7 & 74.2 & 190 & 78 & 128 \\
\hline White star & 61.2 & 109.3 & 26.9 & 19.7 & 76.0 & 182 & 84 & 119 \\
\hline WS_SSi-2 & 73.7 & 95.4 & 30.8 & 22.5 & 62.6 & 145 & 33 & 130 \\
\hline WS_SSi-5 & 68.8 & 96.7 & 30.4 & 20.7 & 63.0 & 139 & 38 & 135 \\
\hline WS_SSi-12 & 74.9 & 98.4 & 27.1 & 19.9 & 63.3 & 144 & 34 & 135 \\
\hline WS_SSi-28 & 78.5 & 61.3 & 27.9 & 18.5 & 62.7 & 164 & 42 & 199 \\
\hline WS_SSi-34 & 86.6 & 69.9 & 25.0 & 17.5 & 60.4 & 141 & 47 & 144 \\
\hline WS_SSi-39 & 70.1 & 100.4 & 28.8 & 19.4 & 64.7 & 126 & 36 & 145 \\
\hline
\end{tabular}

All plants were grown under temperature controlled greenhouse during Aug 6 to Oct 22. a) g fresh weight/plant, b) \% to total fresh weight of storage root, c) \% of total fresh weight based on freeze-drying, d) RVA properties were determined on $7 \%$ starch suspension by Rapid Visco Analyzer. 
Table 2. Physiological trait and RVA starch properties of transgenic sweetpotato plants (cv. Konahomare).

\begin{tabular}{|c|c|c|c|c|c|c|c|c|}
\hline Plant & $\begin{array}{c}\text { Weigt of } \\
\text { aerial part }\end{array}$ & $\begin{array}{c}\text { Storage root } \\
\text { weight }^{\text {a) }}\end{array}$ & $\begin{array}{c}\text { Dry matter } \\
\text { content }^{\mathrm{b}, \mathrm{c})}\end{array}$ & $\begin{array}{c}\text { Starch } \\
\text { content }^{\mathrm{b})}\end{array}$ & $\begin{array}{c}\text { Pasting } \\
\text { temp. }\left({ }^{\circ} \mathrm{C}\right)^{\mathrm{d})}\end{array}$ & $\begin{array}{c}\text { Peak } \\
\text { visc. }(\text { RVU) } \\
\text { d) }\end{array}$ & $\begin{array}{c}\text { Breakdown } \\
(\text { RVU) })^{d}\end{array}$ & $\begin{array}{l}\text { Setback } \\
(\text { RVU) }\end{array}$ \\
\hline Konahomare & 45.1 & 74.3 & 29.5 & 23.7 & 76.0 & 172 & 85.0 & 130 \\
\hline Konahomare & 46.4 & 54.2 & 29.0 & 22.7 & 78.4 & 148 & 49.0 & 127 \\
\hline Konahomare & 59.0 & 72.1 & 28.6 & 22.4 & 77.0 & 163 & 67.0 & 122 \\
\hline Konahomare & 48.2 & 55.7 & 28.2 & 21.9 & 76.7 & 152 & 54.0 & 128 \\
\hline KH_SSi-1 & 30.0 & 55.5 & 32.3 & 24.5 & 63.9 & 104 & 15.0 & 170 \\
\hline KH_SSi-2 & 61.0 & 55.7 & 31.8 & 25.8 & 63.9 & 87 & 1.0 & 111 \\
\hline KH_SSi-3 & 45.5 & 57.4 & 30.2 & 20.8 & 62.0 & 120 & 12.0 & 185 \\
\hline KH_SSi-6 & 59.7 & 71.8 & 30.9 & 20.9 & 62.6 & 131 & 12.0 & 177 \\
\hline KH_SSi-8 & 38.2 & 59.1 & 31.4 & 25.9 & 64.0 & 118 & 5.0 & 172 \\
\hline KH_SSi-9 & 56.0 & 76.0 & 31.7 & 26.9 & 63.7 & 114 & 22.0 & 154 \\
\hline KH_SSi-11 & 58.5 & 78.9 & 32.5 & 22.9 & 63.0 & 129 & 11.0 & 180 \\
\hline KH_SSi-13 & 49.2 & 45.1 & 32.4 & 23.2 & 63.6 & 129 & 11.0 & 175 \\
\hline KH_SSi-15 & 41.8 & 61.2 & 31.8 & 26.1 & 62.3 & 110 & 23.0 & 161 \\
\hline KH_SSi-19 & 52.0 & 68.3 & 32.8 & 26.7 & 64.2 & 125 & 26.0 & 140 \\
\hline KH_SSi-23 & 55.3 & 72.7 & 31.7 & 26.3 & 62.6 & 112 & 11.0 & 156 \\
\hline Quick Sweet & 71.3 & 46.5 & 28.2 & 18.2 & 58.9 & 147 & 18.0 & 156 \\
\hline Quick Sweet & 46.3 & 27.5 & 26.2 & 19.9 & 58.3 & 132 & 11.0 & 146 \\
\hline Quick Sweet & 59.0 & 28.3 & 29.6 & 21.6 & 59.9 & 137 & 9.0 & 162 \\
\hline
\end{tabular}

All plants were grown under temperature controlled greenhouse as same as Table 1 except the growth period during May 7 to July 16 . a-d) All units and experimental condition are same as those in Table 1.

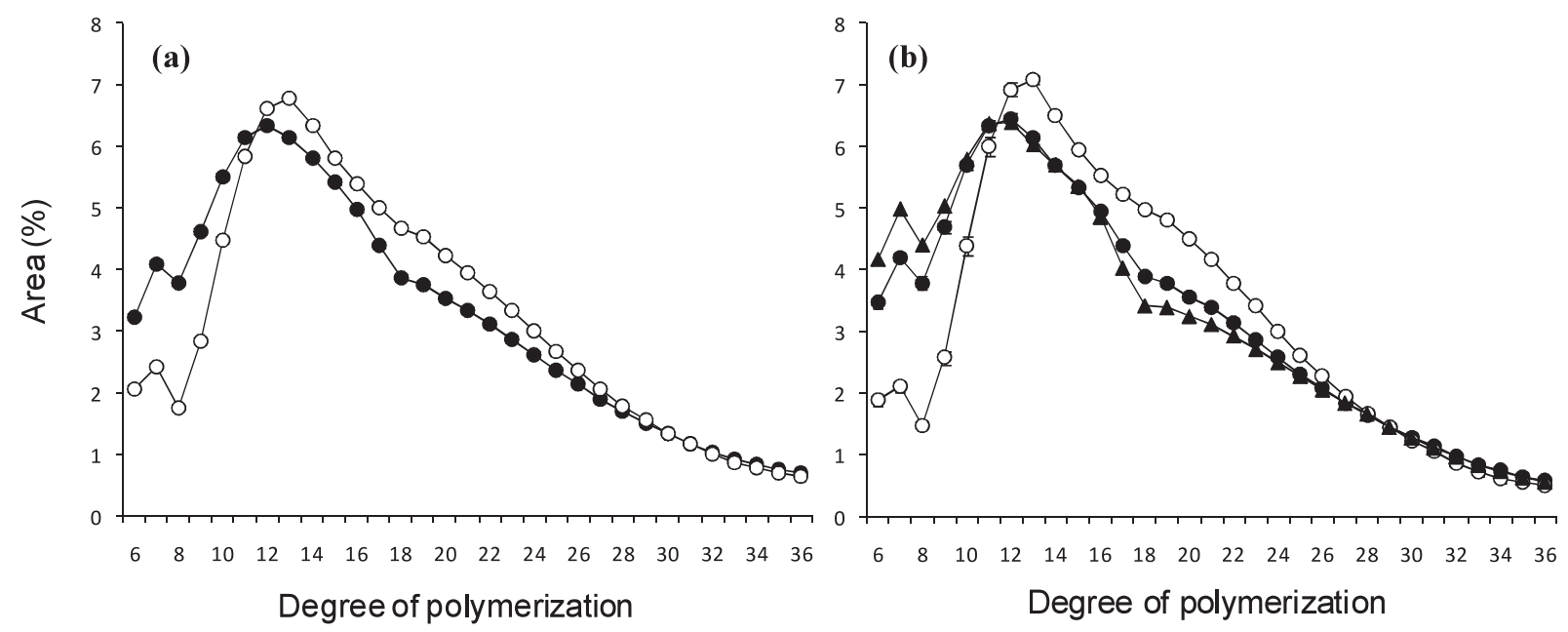

Fig. 3. Profiles of short chain distribution of amylopectin of transgenic (filled circle) and wild-type (opened circle) sweetpotato.

(a) Four transgenic (WS_SSi-2, 5, 12, 39) and 3 wild-type plants of cv. White Star and (b) 4 transgenic (KH_SSi-1, 2, 3, 6), 2 wildtype plants of cv. Konahomare and 2 plants of cv. Quick Sweet (filled triangle) were analyzed and the data were averaged. Error bars show the standard deviation.

fied, may also be associated. These results indicate that SSII bound to the starch granule likely results in alteration of the amylopectin structure in the sweetpotato.

To assess the physiological impact of reduced SSII expression on starch properties in sweetpotato storage roots, transgenic sweetpotato plants with reduced expression of the SSII gene were generated using RNA interference. The cultivars White Star ${ }^{11)}$ and Konahomare were used as transformation materials. The transgenic plants grown in a temperature-controlled green house were harvested, and the physiological traits and Rapid Visco Analyzer (RVA) properties of starch in the storage roots were investigated. Dry matter content and starch yield in the storage roots of these transgenic plants were comparable to those of the wild-type plants, suggesting that starch productivity in these transgenic plants is not affected by suppressing SSII expression (Tables 1 and 2). All starches from transgenic plants showed lower pasting temperatures and reduced breakdown measured by a RVA. Pasting temperatures of starch from transgenic plants were approximately $10-15^{\circ} \mathrm{C}$ lower than those in wild-type plants (Tables 1 and 2) and $3-5^{\circ} \mathrm{C}$ higher than those in QS (Table 2). Distribution of the amylopectin chain length of the transgenic lines showed marked differences from those in wild-type plants: more chains with a DP of 6-11 and fewer chains with a DP of 13-25 (Figs. 3(a) and (b)), although to a lesser extent than that in QS (Fig. 3(b)). We hypothesize that the extent of changes in pasting temperatures and amylopectin chain length distribution were not comparable between transgenic sweetpotatoes and QS due to an insufficient effect of RNA interference of SSII. ${ }^{11)}$ However, this hypothesis remains to be tested.

In conclusion, our results clearly indicate that SSII in sweetpotato storage roots, like that in other plants, affects the pasting properties of starch through alteration of the amylopectin structure.

Acknowledgements: We are grateful to Prof. Alison Smith and Prof. Kazufumi Yazaki for kindly providing the antibody against pea embryo SSII and the hygromycin resistant gene, respectively. We also thank 
Prof. Yasunori Nakamura and Dr. Naoko Fujita for their valuable support throughout this work.

\section{REFERENCES}

1 ) K. Katayama, K. Komae, K. Kohyama, T. Kato, S. Tamiya and K. Komaki: New sweetpotato line having low gelatinization temperature and altered starch structure. Staerke, 54, 51-57 (2002).

2 ) K. Katayama, S. Tamiya, T. Kuranouchi, K. Komaki and M. Nakatani: New sweetpotato cultivar "Quick Sweet". Bull. Nat. Inst. Crop Sci., 3, 35-52 (2003).

3 ) T. Murata and T. Akazawa: Enzymic mechanism of starch synthesis in sweetpotato roots. I. Requirement of potassium ions for starch synthase. Arch. Biochem. Biophys., 126, 873-879 (1968).

4 ) T. Murata: Enzymic mechanism of starch synthesis in sweetpotato roots. Part III. The composition of carbohydrates and soluble nucleotides in the developing sweetpotato roots. Nogei Kagaku Kaishi, 44, 412-421 (1970).

5 ) S.J. Wang, K.W. Yeh and C.Y. Tsai: Molecular characterization and expression of starch granule-bound starch synthase in the sink and source tissues of sweetpotato. Physiol. Plant., 106, 253261 (1999).

6 ) T. Kimura, O. Ideta and A. Saito: Identification of the gene encoding granule-bound starch synthase I in sweetpotato (Ipomoea batatas (L.) Lam.). Plant Biotechnol., 17, 247-252 (2000).

7 ) T. Kimura, M. Otani, T. Noda, O. Ideta, T. Shimada and A. Saito: Absence of amylose in sweetpotato [Ipomoea batatas (L.) Lam.] following the introduction of granule-bound starch synthase I cDNA. Plant Cel. Rep., 20, 663-666 (2001).

8 ) T. Noda, T. Kimura, M. Otani, O. Ideta, T. Shimada, A. Saito and I. Suda: Physicochemical properties of amylose-free starch from transgenic sweetpotato. Carbohydr. Polym., 49, 253-260 (2002).

9 ) M. Otani, T. Hamada, K. Katayama, K. Kitahara, S.H. Kim, Y. Takahata, T. Suganuma and T. Shimada: Inhibition of the gene expression for granule-bound starch synthase I by RNA interference in sweetpotato plants. Plant Cell Rep., 26, 1801-1807 (2007).

10) K. Kitahara, K. Hamasuna, K. Nozuma, M. Otani, T. Hamada, T. Shimada, K. Fujita and T. Suganuma: Physicochemical properties of amylose-free and high-amylose starches from transgenic sweetpotatoes modified by RNA interference. Carbohydr. Polym., 69, 233-240 (2007).

11) Y. Takahata, M. Tanaka, M. Otani, K. Katayama, K. Kitahara, O. Nakayachi, H. Nakayama and M. Yoshinaga: Inhibition of the expression of the starch synthase II gene leads to lower pasting temperature in sweetpotato starch. Plant Cell Rep., 29, 535-543 (2010).

12) I.J. Tetlow, K.G. Beisel, S. Cameron, A. Makhmoudova, F. Liu, N.S. Bresolin, R. Wait, M.K. Morell and M.J. Emes: Analysis of protein complexes in amyloplasts reveals functional interactions among starch synthetic enzymes. Plant Physiol., 146, 1878-1891 (2008).

13) T.A. Hennen-Bierwagen, F. Liu, R.S. Marsh, S. Kim, Q. Gan, I.J. Tetlow, M.J. Emes, M.G. James and A.M. Myers: Starch biosynthetic enzymes from developing maize endosperm associate in multisubunit complexes. Plant Physiol., 146, 1892-1908 (2008).

*E-mail: ytaka@affrc.go.jp

$* * * * * * * * * *$

\section{Omics-based Approach for Cereal Starch Biosynthesis: Towards Determination of Key Factors for Quality of Rice Grain Affected by High Temperature}

\author{
Hiromoto Yamakawa ${ }^{1, *}$ \\ ${ }^{1}$ National Agricultural Research Center, Hokuriku \\ (1-2-1 Inada, Joetsu, Niigata 943-0193, Japan)
}

High temperature impairs grain filling of rice by inhibiting starch accumulation in the endosperm, causing the grain to have a chalky appearance. This affects the molecular structure of a starch component, amylopectin (e.g., elongation of its chains), thereby hardening the texture of cooked rice. Recent comprehensive analyses such as transcriptome, proteome and metabolome, which are assisted by rice genome information, revealed key metabolic factors involved in deterioration of rice grains by exposure to elevated temperatures. ${ }^{1-3)}$ Shortage of starch (defect in starch quantity) may be caused by a combination of decreased expression of starch biosynthesis-related genes such as GBSSI and $B E I I b$ and increased degradation of starch by induced expression of $\alpha$-amylase genes. ${ }^{1)}$ Determination of carbohydrate metabolites further suggested that sugar metabolism and ATP production is also impaired at high temperatures, leading to decreased starch deposition. ${ }^{2)}$ For the hardening of the texture (defect in starch quality), decreased branching of amylopectin by reduced expression of starch branching enzyme genes, particularly short chain-producing BEIIb, may contribute to enrichment of its long chains. ${ }^{1)}$ The effects of high temperatures on grain filling-related metabolism are summarized at the gene level.

\section{REFERENCES}

1 ) H. Yamakawa, T. Hirose, M. Kuroda and T. Yamaguchi: Comprehensive expression profiling of rice grain filling-related genes under high temperature using DNA microarray. Plant Physiol., 144, 258-277 (2007).

2 ) H. Yamakawa and M. Hakata: Atlas of rice grain filling-related metabolism under high temperature: Joint analysis of metabolome and transcriptome demonstrated inhibition of starch accumulation and induction of amino acid accumulation. Plant Cell Physiol., 51, 795-809 (2010).

3 ) H. Yamakawa, T. Ebitani and T. Terao: Comparison between locations of QTLs for grain chalkiness and genes responsive to high temperature during grain filling on the rice chromosome map. Breed. Sci., 58, 337-343 (2008).

* E-mail: hy741220@affrc.go.jp

$* * * * * * * * * *$

\section{Characterization of a Novel Enzyme Activity Involved in Semi-amylopectin Biosynthesis from the Marine Cyanobacterium Clg1}

\author{
Christophe Colleoni, ${ }^{1, *}$ Ugo Cenci, ${ }^{1}$ Cathy Tirtiaux, ${ }^{1}$ \\ Jenifer Nirmal Raj, ${ }^{1}$ Eiji Suzuki, ${ }^{2}$ \\ Yasunori Nakamura ${ }^{2}$ and Steven Ball ${ }^{1}$ \\ ${ }^{1}$ Unité de Glycobiologie Structurale et Fonctionnell \\ (UMR8576 CNRS-USTL 59655 Villeneuve d'Ascq, \\ Cedex, France) \\ ${ }^{2}$ Faculty of Bioresource Science, \\ Akita Prefectural University \\ (Akita 010-0195, Japan)
}

In addition to performing oxygenic photosynthesis, some cyanobacteria are able to reduce atmospheric dinitrogen to ammonia. This enzymatic reaction, catalyzed by the nitrogenase, is extremely sensitive to oxygen and requires a large amount of ATP (16 ATP/N2). Nitrogen fixing cyanobacteria also called diazotrophic cyanobacteria have developed several strategies to circumvent the incompatibility between nitrogen fixation and oxygenic photosynthesis. In aerobic filamentous cyanobacteria, nitrogenase activity is localized in specialized cells named heterocysts that lack the O2-evolving photosystem II. Thus, the energy needed for 
nitrogen fixation in heterocysts is supplied by neighboring cells during the light period. In contrast to heterocystforming cyanobacteria, aerobic unicellular cyanobacteria achieve temporal confinement of oxygenic photosynthesis, which occurs naturally during the day, whereas nitrogen fixation is restricted to the night. Interestingly, several unicellular diazotrophic cyanobacteria belonging to group $\mathrm{V}$ have been recently reported to accumulate, semi-crystalline starch-like carbohydrate granules while most other cyanobacteria have been reported to store glycogen. Although there is no direct evidence, high-energy cost of nitrogen fixation process could explain the emergence of novel and more adapted storage polysaccharide in this particular group of unicellular cyanobacteria. In order to investigate this pathway, a UV-mutagenesis campaign was carried out on the unicellular cyanobacteria strain Clg1. Mutants impaired in starch metabolism were isolated by iodine staining of cell patches. We screened over 20,000 cell patches and isolated 90 mutants displaying different iodine-staining phenotypes. Among them, five mutants isolated with different mutagenesis conditions displayed a yellow-orange stain easy to distinguish from the dark blue standard wild-type stain. This is correlated with a low amount of starch $(10 \%$ of residual starch) and an increase of 2 to 5 fold of water-soluble polysaccharide in comparison to wild-type strain. Interestingly, all mutants characterized so far, lack an activity band visualized onto glycogen containing zymograms gels. Although further investigations are required to identify this enzymatic defect, these preliminary experiments suggest that this starch-metabolizing enzyme is involved in a critical step of starch biosynthesis in the marine cyanobacterium Clg1.

\section{* E-mail: Christophe.colleoni@univ-lille1.fr} $* * * * * * * * * *$

\section{Characterization of Cyanobacterial Starch}

\author{
Eiji Suzuki, ${ }^{1, *}$ Miho Onoda, ${ }^{1}$ Christophe Colleoni, ${ }^{2}$ \\ Steven Ball, ${ }^{2}$ Naoko Fujita ${ }^{1}$ and Yasunori Nakamura ${ }^{1}$ \\ ${ }^{1}$ Faculty of Bioresource Sciences, \\ Akita Prefectural University \\ (241-438 Kaidobata-nishi, Shimoshinjo-nakano, \\ Akita 010-0195, Japan) \\ ${ }^{2}$ Unité de Glycobiologie Structurale et Fonctionnell \\ (UMR8576 CNRS-USTL 59655 Villeneuve d'Ascq, \\ Cedex, France)
}

The phrase "cyanobacterial starch" has been used occasionally in a casual context, referring to the storage polysaccharides in cyanobacteria. However, the storage polysaccharide in most cyanobacterial species is glycogen. In contrast, a starch-like polysaccharide has been recently reported ${ }^{1)}$ and its occurrence is confined to unicellular diazotrophic species within the group $\mathrm{V}$ of cyanobacteria. The distinction between cyanobacterial glycogen and starch is straightforward: when the cells are disrupted and centrifuged, glycogen remains soluble in the supernatant, while starch becomes immediately visible as a white precipitate at the bottom of the centrifuge tube. When first described by Nakamura and colleagues, the only information available regarding cyanobacterial starch was its higher molecular mass (as compared to that of glycogen) and unique pattern of chain length distribution (presence of very short chains of DP 4-5; most abundant chains being DP 11-12, highly analogous to those in rice amylopectin, and relatively small amounts of long chains of $\mathrm{DP} \geq 37$ ). On the basis of these characteristics, the polysaccharide was considered to be an intermediate between glycogen and amylopectin, and, therefore, designated as "semi-amylopectin." We conducted further analyses using purified preparations of polysaccharides from 3 strains, and observed that Cyanobacterium sp. NBRC 102756 (formerly MBIC 10216), Cyanothece sp. ATCC 51142, and the unclassified strain CLg1, and showed additional features, some of which are very much like those of amylopectin in plants. Common characteristics as well as variability observed with these polysaccharide preparations are discussed.

\section{REFERENCES}

1 ) Y. Nakamura, J. Takahashi, A. Sakurai, Y. Inaba, E. Suzuki, S. Nihei, S. Fujiwara, M. Tsuzuki, H. Miyashita, H. Ikemoto, M. Kawachi, H. Sekiguchi, N. Kurano: Some cyanobacteria synthesize semi-amylopectin type $\alpha$-polyglucans instead of glycogen. Plant Cell Physiol., 46, 539-545(2005).

*E-mail: esuzuki@akita-pu.ac.jp $* * * * * * * * * *$

\section{The Effect of Starch Molecular Structures on Starch Hydrothermal and Degradative Properties of Potatoes}

\author{
Takahiro Noda, ${ }^{1, *}$ Hetti Arachchige Mangalika \\ Wickramasinghe ${ }^{2}$ and Andreas Blennow ${ }^{3}$ \\ ${ }^{1}$ National Agricultural Research Center \\ for Hokkaido Region \\ (Memuro, Hokkaido 082-0081, Japan) \\ ${ }^{2}$ Department of Agricultural Biology, \\ Faculty of Agriculture, University of Peradeniya \\ (Peradeniya, 20400, Sri Lanka) \\ ${ }^{3}$ VKR Centre for Pro-Active Plants, \\ Department of Plant Biology and Biotechnology, \\ Faculty of Life Sciences, University of Copenhagen \\ (40 Thorvaldsensvej DK-1871 Frederiksberg C, \\ Copenhagen, Denmark)
}

Starch phosphorylation is the only naturally occurring modification of starch. The level of starch phosphorylation varies with the botanical source and it was shown that starches from tuberous tissues are more phosphorylated than those of cereal seeds. ${ }^{1,2)}$ Potato, the most extensively studied system with respect to starch phosphorylation, is known to contain relatively large amounts of covalently bound phosphate. It is now well established that reduced activity of starch branching enzymes (SBE) by antisense gene technology increases the content of longer amylopectin chains and increases starch phosphorylation., ${ }^{3,4)}$ Suppression of the granule bound starch synthase (GBSS) generates a virtually amylose-free tuber starch but only results in a minor increase in phosphate levels which is an effect of the decreased amylose content, while amylose is not phosphorylated. Furthermore, starch phosphorylation at the C-6 position of glucose residues is catalyzed by glucan water dikinase (GWD). ${ }^{6}$

In this study, we used starches re-structured directly in 
Table 1. Coefficient of variance for different starch properties as calculated for amylose content, G6P content and amylopectin average DP.

\begin{tabular}{lccc}
\hline \multicolumn{1}{c}{ Starch property } & $\begin{array}{c}\text { Amylose } \\
\text { content }\end{array}$ & G6P & $\begin{array}{c}\text { Average } \\
\text { DP }\end{array}$ \\
\hline Amylose content & - & $0.61^{*}$ & $0.60^{*}$ \\
G6P & - & - & $0.97^{* * *}$ \\
Enzyme digestibility of raw starch & $-0.22^{\mathrm{NS}}$ & $-0.75^{* *}$ & $-0.77^{* * *}$ \\
Peak viscosity & $0.21^{\mathrm{NS}}$ & $0.67^{* *}$ & $0.70^{* *}$ \\
Breakdown viscosity & $-0.37^{\mathrm{NS}}$ & $0.20^{\mathrm{NS}}$ & $0.28^{\mathrm{NS}}$ \\
Setback & $0.89^{* * *}$ & $0.72^{* *}$ & $0.67^{* *}$ \\
Peak gelatinization temperature & $0.45^{\mathrm{NS}}$ & $0.91^{* * *}$ & $0.89^{* * *}$ \\
Onset gelatinization temperature & $0.45^{\mathrm{NS}}$ & $0.94^{* * *}$ & $0.88^{* * *}$ \\
Enthalpy for starch gelatinization & $-0.75^{* * *}$ & $-0.55^{*}$ & $-0.53^{*}$ \\
\hline
\end{tabular}

*, ** and *** Significant at the $P \leq 0.05, P \leq 0.01$ and $P \leq 0.001$, respectively. ${ }^{\text {NS }}$ not significant.

potato tubers by anti-sense suppression of SBE, GBSS, or GWD genes to analyze starch molecular structures, amylose, covalently esterified glucose-6-phosphate (G6P) contents, and the amylopectin average degree of polymerization (DP). Potato starches were also analyzed for pasting properties, peak viscosity, breakdown viscosity, and setback using Rapid Visco Analysis, thermal properties, peak and onset gelatinization temperatures, and enthalpy for starch gelatinization using differential scanning calorimetry and enzyme digestibility of raw starch using glucoamylase.

Compared to wild-type potatoes, transgenic potatoes with suppressed SBE, GBSS and GWD activities resulted in dramatic changes in starch molecular structure as well as significant variations in pasting and thermodynamic properties. As a result, the digestibility with glucoamylase was altered. Correlation coefficients were calculated to evaluate the effect of starch molecular structures on starch hydrothermal and degradative properties in 15 potato starches (Table 1). Despite extreme alterations in the G6P content, the amylose content had a larger influence on the enthalpy of starch gelatinization. Interestingly, the G6P content, in addition to the average DP of amylopectin chains, exhibited a higher correlation than amylose to peak and onset gelatinization temperatures of starch. The G6P content and amylopectin chain length controlled the peak viscosity and setback. Clear inverse correlations between glucoamylase catalyzed digestibility and amylopectin chain length and starch phosphate; this lack of correlation with amylose content indicates a combined structuring role of the phosphate groups and amylopectin chains on the starch glucan matrix.

\section{REFERENCES}

1 ) A. Blennow, S.B. Engelsen, L. Munck and B.L. Møller: Starch molecular structure and phosphorylation investigated by a combined chromatographic and chemometric approach. Carbohydr. Polym., 41, 163-174 (2000).

2 ) S. Tabata, K. Nagata and S. Hizukuri: Studies on starch phosphates. Part 3. On the estimated phosphates in some cereal starches. Starch/Stärke, 27, 333-335 (1975).

3 ) A. Blennow, B. Wischmann, K. Houborg, T. Ahmt, K. Jorgensen, S.B. Engelsen, O. Bandsholm and P. Poulsen: Structure function relationships of transgenic starches with engineered phosphate substitution and starch branching. Int. J. Biol. Macromol., 36, 159-168 (2005).

4 ) G.P. Schwall, R. Safford, R.J. Westcott, R. Jeffcoat, A. Tayal, Y.C. Shi, M.J. Gidley and S.A. Jobling: Production of very-highamylose potato starch by inhibition of SBE A and B. Nat. Biotechnol., 18, 551-554 (2000).

5 ) S.S. Kozlov, A. Blennow, A.V. Krivandin and V.P. Yuryev: Struc- tural and thermodynamic properties of starches extracted from GBSS- and GWD suppressed potato lines. Int. J. Biol. Macromol., 40, 449-460 (2007).

6 ) G. Ritte, M. Heydenreich, S. Mahlow, S. Haebel, O. Kötting and M. Steup: Phosphorylation of C6- and C3-positions of glucosyl residues in starch is catalyzed by distinct dikinases. FEBS Lett., 580, 4872-4876 (2006).

*E-mail: noda@affrc.go.jp

$* * * * * * * * * *$

\section{Increased Biomass and Yield in Wheat Results from Down Regulation of Glucan Water Dikinase Activity}

\author{
Jean-Philippe Ral, ${ }^{1}$ Andrew F. Bowerman, ${ }^{1,3}$ \\ Zhongyi Li, ${ }^{1}$ Xavier Sirault, ${ }^{2}$ Robert Furbank, ${ }^{2}$ \\ Jenifer R. Pritchard, ${ }^{1}$ Marianne Bloemsma, ${ }^{1}$ \\ Colin R. Cavanagh, ${ }^{1}$ Crispin A. Howitt ${ }^{1}$ \\ and Matthew K. Morell ${ }^{1, *}$ \\ ${ }^{1}$ CSIRO Food Futures National Research Flagship \\ (GPO Box 1600, Canberra ACT 2601 Australia) \\ ${ }^{2}$ CSIRO Plant Industry \\ (GPO Box 1600, Canberra ACT 2601 Australia) \\ ${ }^{3}$ School of Biochemistry \& Molecular Biology, \\ The Australian National University, Canberra \\ (ACT 0200, Australia)
}

Wheat starch has a low intrinsic pasting viscosity relative to other available starches. Consequently wheat starch is less competitive for many applications to starches from potato, tapioca and waxy maize because it produces less viscous pastes on heating in water. In this work, we sought to modulate wheat starch phosphate content as a mechanism to test the hypothesis that pasting viscosity could be modulated by modifying starch phosphate content.

Glucan-water dikinase (GWD) is known to be responsible for starch phosphorylation in plant and in particularly in transitory starch metabolism. GWD catalyzes the transfer of a phosphate group from ATP to the 6 hydroxyl position of glucosyl units within pre-existing glucan chains. The phosphate group is proposed to be the target signal for enzymes such as $\alpha$-amylases or isoamylases to localise and begin starch degradation through localised disordering of starch crystallites. Mutations of the GWD leads to a starch excess phenotype in Arabidopsis thaliana.

While the involvement of GWD in circadian starch metabolism is well established, however, little is known regarding the role of GWD in reserve starch, and in particular, in the cereal endosperm.

Endosperm specific RNAi mediated down-regulation GWD in wheat resulted in a reduction of GWD expression and a decrease in starch phosphate content in the endosperm. Surprisingly an increase in vegetative biomass and grain yield was also observed. Effects on plant growth and development were seen from the 2 leaf stage. Yield increases were constantly obtained in both pot and plot trials in glasshouses. In pots, grain yield per plant increased across all GWD inactivation lines by $29 \%$. In simulated plot trials, an average yield of $26 \%$ was observed for GWD inactivation lines compared to control. The enhanced yield resulted from a combination of increases in spikelets per head, seed number per spike and seed weight. In addition an increase in stem 
carbohydrate levels and increased levels of $\alpha$-amylase 3 activity in the aleurone layer of the grain were observed. No changes were seen in the activity of other enzymes involved in starch degradation assayed. These findings provide a potentially important novel mechanism to increase yield and modify grain quality in crop improvement programs.

* E-mail: Matthew.Morell@csiro.au $* * * * * * * * * *$

\section{Diversity of Function and Structure of Isoamylase}

\author{
Yoshinori Utsumi ${ }^{1, *}$ and Yasunori Nakamura ${ }^{1}$ \\ ${ }^{1}$ Faculty of Bioresource Science, Akita Prefectural University \\ (Akita 010-0195, Japan)
}

Isoamylase (ISA) is involved in starch biosynthesis as ISA1-ISA2 hetero-oligomer in potato tuber ${ }^{1)}$ and Arabidopsis leaves, 2,3) where both proteins exist only as heterooligomers. In contrast, the endosperm of rice and maize contain both the ISA1 homo-oligomer and the ISA1-ISA2 hetero-oligomer. ${ }^{4,5)}$ Although the mechanism by which ISAs are involved in amylopectin synthesis is unclear, it is thought that these enzymes play a crucial role in amylopectin biosynthesis by removing excess branches ${ }^{6)}$ or improper branches ${ }^{7)}$ that interfere with formation of double helices of amylopectin cluster chains ${ }^{7)}$ and starch crystallization. ${ }^{8}$

Recently, Kubo et al. ${ }^{5)}$ revealed that either the homo- or hetero-oligomer is competent for starch biosynthesis in maize endosperm on the basis of results obtained using maize ISA1 and ISA2 mutants. However, the phenotypes resulting from lesions of the ISA1 or ISA2 gene and the hypothesized functions of both ISA proteins in starch synthesis differs between maize and rice endosperm. The present comprehensive studies ${ }^{9}$ using protein-based analyses of ISA oligomers from a variety of rice transgenic lines may provide further insight into the functions and structures of both oligomers in plant tissues. To examine the contribution of the ISA1 homo-oligomer and ISA1-ISA2 hetero-oligomer on starch synthesis, expression of the ISA1 or ISA2 were differentially regulated in various transgenic rice plants. Although suppression of ISA2 caused the endosperm to contain only the homo-oligomer, no significant effects were detected regarding starch phenotypes. In contrast, ISA2 over-expression led to the endosperm containing only the hetero-oligomer, and starch synthesis in the endosperm was significantly impaired, both quantitatively and qualitatively, because the starch was devoid of typical starch features, e.g., thermal and radiographic diffraction properties and accumulation of water-soluble highly branched malto-dextrins. In the ISA2 over-expressing line, about $60-70 \%$ of the ISA1-ISA2 hetero-oligomer was bound to starch, while the ISA homo- and hetero-oligomers from wild-type were mostly present in the soluble form at the early-milking stage of the endosperm. Detailed analysis of the relative amounts of homo- and hetero-oligomers in various lines also led to the conclusion that the ISA1 homo-oligomer, but not the ISA1-ISA2 oligomer, is essential, for starch production in the rice endosperm. The relative amounts of ISA1 and ISA2 proteins were shown to impact the ratio of both oligomers and the stoichiometry of both ISAs in the hetero-oligomer.
In addition, the hetero-oligomer was found to be composed of a mixture of heteromers with different ISA1/ISA2 compositions in transgenic as well as wild-type lines. We also found that rice leaves contained only the ISA1-ISA2 heteromer, playing an essential role in normal amylopectin synthesis. When compared with the homo-oligomer, all hetero-oligomers from rice endosperm, leaves, and potato tuber were significantly more stable at $40^{\circ} \mathrm{C}$. Thus, the present investigation ${ }^{9)}$ clearly shows that the structure of the functional ISA oligomer is markedly different between the plant species and tissues.

\section{REFERENCES}

1 ) H. Hussain, A. Mant, R. Seals, S. Zeeman, E. Hinchliffe, A. Edwards, C. Hylton, S. Bornemann, A.M. Smith, C. Martin and R. Bustos: Three isoforms of isoamylase contribute different catalytic properties for the debranching of potato glucans. Plant Cell, 15, 133-149 (2003).

2 ) T. Delatte, M. Trevisan, M.L. Parker and S.C. Zeeman: Arabidopsis mutants Atisa1 and Atisa2 have identical phenotypes and lack the same multimeric isoamylase, which influences the branch point distribution of amylopectin during starch synthesis. Plant J., 41, 815-830 (2005).

3 ) F. Wattebled, Y. Dong, S. Dumez, D. Delvalle, V. Planchot, P. Berbezy, D. Vyas, P. Colonna, M. Chatterjee, S. Ball and C. D'Hulst: Mutants of Arabidopsis lacking a chloroplastic isoamylase accumulate phytoglycogen and an abnormal form of amylopectin. Plant Physiol., 138, 184-195 (2005).

4 ) Y. Utsumi and Y. Nakamura: Structural and enzymatic characterization of the isoamylase1 homo-oligomer and the isoamylase1isoamylase2 hetero-oligomer from rice endosperm. Planta, 225, 75-87 (2006).

5 ) A. Kubo, C. Colleoni, J.R. Dinges, Q. Lin, R.R. Lappe, J.G. Rivenbark, A.J. Meyer, S.G. Ball, M.G. James, T.A. HennenBierwagen and A.M. Myers: Functions of heteromeric and homomeric isoamylase-type starch-debranching enzymes in developing maize endosperm. Plant Physiol., 153, 956-969 (2010).

6 ) S. Ball, H.P. Guan, M. James, A. Myers, P. Keeling, G. Mouille, A. Buléon, P. Colonna and J. Preiss: From glycogen to amylopectin: a model for the biogenesis of the plant starch granule. Cell, 86, 349-352 (1996).

7 ) Y. Nakamura Y: Towards a better understanding of the metabolic system for amylopectin biosynthesis in plants: rice endosperm as a model tissue. Plant Cell Physiol., 43, 718-725 (2002).

8 ) A.M. Myers, M.K. Morell, M.G. James and S.G. Ball: Recent progress toward understanding biosynthesis of the amylopectin crystal. Plant Physiol., 122, 989-997 (2000).

9 ) Y. Utsumi, C. Utsumi, T. Sawada, N. Fujita and Y. Nakamura: Functional diversity of isoamylase (ISA) oligomers: the ISA1 homo-oligomer is essential for amylopectin biosynthesis in rice endosperm. Plant Physiol., 156, 61-77 (2011).

* E-mail: utsumiy@psc.riken.jp $* * * * * * * * * *$

\section{Maize SSIII Affects Activity of Specifically the Homomeric Form of Isoamylase-type Starch Debranching Enzyme}

\author{
Alan Myers ${ }^{1, *}$ \\ ${ }^{1}$ Department of Biochemistry, Biophysics, \\ and Molecular Biology, Iowa State University \\ (Ames, Iowa 50011, USA)
}

Starch synthatse SSIII is one of only two enzymes known to condition accumulation of phytoglycogen in maize endosperm, the other being isoamylase-type starch debranching enzyme (ISA). SSIII mutations alone do not cause this 
so-called "sugary" phenotype, however, when the ISA1 protein is compromised by the su1-P mutation, the gene du1 that encodes SSIII becomes a determining factor in the ability to normally produce crystalline starch. In this study SSIII was also shown to also condition phytoglycogen accumulation in lines lacking a functional ISA2 protein. Endosperm tissue in lines completely lacking both SSIII and ISA2 fail to display any ISA activity in zymograms, however, the ISA homomer complex does assemble and migrate normally in anion exchange chromatography, gel permeation chromatography, and native-PAGE. Thus, SSIII affects the activity of homomeric ISA without influencing it's assembly. Coupling the du1-Ref mutation, which produces a low level of a trunctated SSIII protein, with the isa2 mutation also caused a sugary phenotype. In this instance the ISA homomer did display activity in zymograms. These data may be explained by a direct or indirect effect in which SSIII influences the post translational modification of assembled ISA1 homomer and thus affects its enzymatic activity. This activity of SSIII appears is required for normal starch accumulation if the ISA1/ISA2 heteromer is present.

* E-mail: ammyers@iastate.edu 